\title{
MODULES OF CONSTANT JORDAN TYPE
}

\author{
JON F. CARLSON*, ERIC M. FRIEDLANDER* AND JULIA PEVTSOVA*
}

\begin{abstract}
We introduce the class of modules of constant Jordan type for a finite group scheme $G$ over a field $k$ of characteristic $p>0$. This class is closed under taking direct sums, tensor products, duals, Heller shifts and direct summands, and includes endotrivial modules. It contains all modules in an Auslander-Reiten component which has at least one module in the class. Highly non-trivial examples are constructed using cohomological techniques. We offer conjectures suggesting that there are strong conditions on a partition to be the Jordan type associated to a module of constant Jordan type.
\end{abstract}

\section{Contents}

0. Introduction

1. Constant Jordan Type

2. Examples of modules of constant Jordan type

3. Specialization, $\Gamma(G)_{M}$, and constant Jordan type 9

4. Behavior with respect to tensor products 13

5. Endotrivial modules 20

6. Constructing modules of constant Jordan type 23

7. Constraint on ranks 34

8. Auslander Reiten Components 35

9. Questions and Conjectures 42

10. APPENDIX: Decomposition of tensor products of $k[t] / t^{p}$-modules 45 References 46

\section{INTRODUCTION}

In [18] and [20, the second and third authors have introduced a seemingly naive approach to the study of representations of finite groups and related structures on vector spaces over a field $k$ of characteristic $p>0$. The basic idea is to restrict representations to certain subalgebras (" $\pi$-points") isomorphic to the group algebra of $\mathbb{Z} / p \mathbb{Z}$, for we completely understand the representation theory of the algebra $k \mathbb{Z} / p \mathbb{Z}$ in terms of partitions (or "Jordan types"). The simplicity of this approach

2000 Mathematics Subject Classification. 16G10, 20C20, 20G10.

* partially supported by the NSF . 
enables the authors to consider representation theory in a very general context (of a finite group scheme $G$ over an arbitrary field of characteristic $p>0$ ) and prove both global results about the stable module category and explicit results for specific examples. The naivety of the approach is somewhat misleading for underlying many theorems are somewhat difficult cohomological results, especially results giving finite generation and detection modulo nilpotence on subalgebras of special form.

In a recent paper [21], the second and third authors in collaboration with Andrei Suslin have adopted this naive point of view to formulate and investigate new invariants for such representations. The authors introduce "maximal" and "generic" Jordan types for a given representation whose existence even in the very special case of the finite group $\mathbb{Z} / p \mathbb{Z} \times \mathbb{Z} / p \mathbb{Z}$ is highly non-trivial.

Indeed, this special example $G=\mathbb{Z} / p \mathbb{Z} \times \mathbb{Z} / p \mathbb{Z}$ (for $p>2$ ) is challenging from a representation-theoretic point of view for its group algebra has wild representation type. With such "wildness" in mind, it is natural to investigate classes of representations of $G$ with special properties. That is the purpose of this present paper, in which we investigate modules of constant Jordan type. Although the formulation of this concept requires the approach of " $\pi$-points" and our study utilizes many of the techniques of the papers mentioned above, the resulting class of modules appears to be a most natural one to study for those considering the modular representation theory of finite groups, $p$-restricted Lie algebras, and other finite group schemes. We remark here on two aspects of this class of modules of constant Jordan type: it includes the much-studied class of endotrivial modules and also includes many other modules even in the special case of an elementary abelian $p$-group; the classification of such modules of constant Jordan type appears to be very difficult, sufficiently difficult that even in the special case $G=\mathbb{Z} / p \mathbb{Z} \times \mathbb{Z} / p \mathbb{Z}$ we can only speculate on what Jordan types are realized.

In Definition 1.5, we introduce the concept of a $k G$-module $M$ of constant Jordan type, a finite dimensional module with the property that $\alpha_{K}^{*}\left(M_{K}\right)$ has Jordan type independent of the $\pi$-point $\alpha_{K}: K[t] / t^{p} \longrightarrow K G$ with $K / k$ an arbitrary field extension. As verified in Theorem 5.6, a $k G$-module is an endotrivial module if and only if it has constant Jordan type of a very special form. For certain explicit finite group schemes, various examples of modules of constant Jordan type can be constructed directly as we show in 92 . Much of our effort in the first half of this paper is dedicated to showing that the class of modules of constant Jordan type is closed under various natural operations: Heller shifts (Proposition 1.8), direct sums (Proposition 1.8), direct summands (Theorem 3.7), linear duals (Proposition 5.2), and tensor products (Corollary 4.3).

To establish these results, we continue the study initiated in [21] of the condition that a $\pi$-point $\alpha_{K}$ be maximal for a given $k G$-module $M$. This analysis should be of interest for the study of general $k G$-modules. In $\S 3$, we formulate the natural relationship of strict specialization of $\pi$-points (closely related to the relationship 
of specialization of equivalence classes of $\pi$-points considered in [20]). In $\S 4$, we investigate the surprisingly subtle behavior of the condition of maximality of $\pi$ points with respect to the tensor product of two given $k G$-modules. The relevance of maximality of $\pi$-points for a $k G$-module $M$ is emphasized by Proposition 3.6 which asserts that the $k G$-module $M$ has constant Jordan type if and only if the non-maximal support variety of $M, \Gamma(G)_{M}$, is empty.

In the second half of this paper, we give several methods of constructing modules of constant Jordan type. One is provided in Proposition 6.1 and another in Theorem 6.6 (as well as Proposition 6.7). Much preliminary effort is required for us to establish in Theorem 6.13 that our second method provides examples which can not be realized by the first. Indeed, the example provided by this theorem shows how subtle is the behavior of the class of modules of constant Jordan type with respect to extensions. A third method of construction using the Auslander-Reiten theory of almost split sequences is detailed in 88 ; Theorem 8.7 establishes that any module in the same Auslander-Reiten component as a module of constant Jordan type is also of constant Jordan type, whereas Theorem 8.8 constructs $k G$-modules of constant Jordan type $n[1]+($ proj) provided that $G$ satisfies a mild cohomological property.

As one indication of the combinatorics involved in the existence of modules of constant Jordan type, we show in Theorem 7.1 that our techniques give a new proof of a special case of Macaulay's Generalized Principal Ideal Theorem.

We have made little progress in classifying those partitions which are realizable as the Jordan type of modules of constant Jordan type. For example, for a rank 2 elementary abelian $p$-group $E$ with $p>3$, we conjecture but can not prove that no partition of type $n[p]+1[2]$ is the Jordan type of $k E$-module of constant Jordan type. In 99 , we mention numerous questions and conjectures on the constraints of a Jordan type associated to a module of constant Jordan type.

Throughout this paper, $k$ will denote an arbitrary field of finite characteristic and $p>0$ will denote the characteristic of $k$. Without explicit mention to the contrary, $k G$-modules are assumed to be finitely generated. We let $M_{n}(k)$ denote the algebra of $n \times n$ matrices over $k$.

We thank Valery Alexeev, David Eisenbud, and Sasha Premet for help with Theorem 7.1. We also thank Karin Erdmann for drawing our attention to possible connections with the Auslander-Reiten theory. We are particularly grateful to the referee for many constructive comments and suggestions.

\section{Constant Jordan Type}

In this first section, we introduce modules of constant Jordan type and investigate some of the basic properties of this class of modules.

Recall that a finite group scheme $G$ (over $k$ ) is a group scheme over $k$ whose coordinate algebra $k[G]$ is finite dimensional over $k$. We denote the linear dual 
of $k[G]$ by $k G$ and call this the group algebra of $G$. A (rational) $G$-module is a comodule for $k[G]$ or equivalently a $k G$-module. If $K / k$ is a field extension, then we denote by $G_{K}$ the base change of the $k$-group scheme $G$ to the $K$-group scheme $G_{K}=G \times_{\text {Spec } k}$ Spec $K$. We observe that the group algebra $K G_{K}$ of $G_{K}$ equals $K G=K \otimes_{k} k G$.

We remind the reader that the isomorphism class of a finite dimensional $k[t] / t^{p_{-}}$ module $M$ of dimension $n$ (over $k$ ) is given by a partition of $n$ into blocks of size $\leq p$. Equivalently, if we let $\rho_{M}: k[t] / t^{p} \longrightarrow M_{n}(k)$ be the representation associated to $M$, then the isomorphism type of $M$ is specified by the conjugacy class of the element $\rho_{M}(t) \in M_{n}(k)$ whose $p$-th power is 0 . We shall often denote the isomorphism type of $M$ by $a_{p}[p]+\cdots+a_{1}[1]$, where $a_{i}$ denotes the number of blocks of size $i$ in the partition of $n$ associated to $M$.

We call the isomorphism type of a finite dimensional $k[t] / t^{p}$-module $M$ the Jordan type of $M$. For any finite dimensional $k[t] / t^{p}$-module $M$, the stable Jordan type of $M$ is the "stable equivalence" class of Jordan types, where two Jordan types $a_{p}[p]+\cdots+a_{1}[1]$ and $b_{p}[p]+\cdots+b_{1}[1]$ are stably equivalent if $a_{i}=b_{i}$, for all $i<p$.

We may view a Jordan type $a_{p}[p]+\cdots+a_{1}[1]$ as a partition of $n=\sum_{i=1}^{p} i a_{i}$. If $\sum i a_{i}=\sum i b_{i}$, then we say that the Jordan type $\underline{a}=a_{p}[p]+\cdots+a_{1}[1]$ is greater or equal to the Jordan type $\underline{b}=b_{p}[p]+\cdots+b_{1}[1]$ (denoted $\underline{a} \geq \underline{b}$ ) provided that

$$
\sum_{i=j}^{p} i a_{i} \geq \sum_{i=j}^{p} i b_{i}, \quad 1 \leq j \leq p .
$$

If $\underline{a} \geq \underline{b}$ and if $\sum_{i=j}^{p} i a_{i}>\sum_{i=j}^{p} i b_{i}$ for some $j$, then we write $\underline{a}>\underline{b}$. Note that this is the usual dominance ordering on partitions.

Remark 1.1. Let $M, N$ be $k[t] / t^{p}$-modules of dimension $n$ given by

$$
\rho_{M}, \rho_{N}: k[t] / t^{p} \longrightarrow M_{n}(k) .
$$

Then the Jordan type $\underline{a}$ of $M$ is greater or equal to (respectively, greater than) the Jordan type of $\underline{b}$ of $N$ if and only if for every $j, 1 \leq j<p$, the rank of $\rho_{M}^{j}$ is greater or equal to the the rank of $\rho_{N}^{j}$ (resp., and strictly greater for some $j$ ).

Definition 1.2. A $\pi$-point for a finite group scheme $G$ is a left flat map of $K$-algebras $\alpha_{K}: K[t] / t^{p} \longrightarrow K G$, for some field extension $K / k$, which factors through the group algebra $K C_{K} \subset K G_{K}$ of some unipotent abelian subgroup scheme $C_{K} \subset G_{K}$. If $M$ is a finite dimensional $k G$-module, the Jordan type of the $\pi$-point $\alpha_{K}$ on $M$ is the isomorphism class of the $K[t] / t^{p}$-module $\alpha_{K}^{*}\left(M_{K}\right)$ (where $M_{K}=K \otimes_{k} M$ ). We emphasize here that $\alpha_{K}^{*}\left(M_{K}\right)$ denotes the restriction of $M_{K}$ to a $K[t] / t^{p}$-module along the map $\alpha_{K}$. We say that the Jordan type of $\alpha_{K}^{*}\left(M_{K}\right)$ is the Jordan type of $\alpha_{K}$ on $M$. 
Definition 1.3. Let $\alpha_{K}: K[t] / t^{p} \longrightarrow K G, \beta_{L}: L[t] / t^{p} \longrightarrow L G$ be $\pi$-points of $G$. Then $\alpha_{K}$ is said to be a specialization of $\beta_{L}$ (written $\beta_{L} \downarrow \alpha_{K}$ ) if for every finite dimensional $k G$-module $M$ the $K[t] / t^{p}$-module $\alpha_{K}^{*}\left(M_{K}\right)$ is projective whenever the $L[t] / t^{p}$-module $\beta_{L}^{*}\left(M_{L}\right)$ is projective. We say that $\alpha_{K}, \beta_{L}$ are equivalent and write $\alpha_{K} \sim \beta_{L}$ provided that $\alpha_{K} \downarrow \beta_{L}$ and $\beta_{L} \downarrow \alpha_{K}$.

The following theorem summarizes the close relationship between the set of equivalence classes of $\pi$-points of $G$ and the cohomology $\mathrm{H}^{\bullet}(G, k)$. Here, $\mathrm{H}^{\bullet}(G, k)=$ $\mathrm{H}^{*}(G, k)$, the cohomology algebra of $G$ provided that $p=2$; for $p>2, \mathrm{H}^{\bullet}(G, k) \subset$ $\mathrm{H}^{*}(G, k)$ denotes the commutative subalgebra of even dimensional classes.

Theorem 1.4. ([20, 3.6]) The set of equivalence classes of $\pi$-points of a finite group scheme $G$, written $\Pi(G)$, admits a scheme structure determined by the stable module category, stmod $(k G)$. With this structure, $\Pi(G)$ is isomorphic to the scheme $\operatorname{Proj} \mathrm{H}^{\bullet}(G, k)$.

In particular, the closed subsets of $\Pi(G)$ are of the form $\Pi(G)_{M}$ where $M$ is a finite dimensional $k G$-module and $\Pi(G)_{M}$ is the subset of those equivalence classes of $\pi$-points $\alpha_{K}: K[t] / t^{p} \longrightarrow K G$ such that $\alpha_{K}^{*}\left(M_{K}\right)$ is not projective.

We now introduce modules of constant Jordan type, whose study is the primary object of interest in this paper.

Definition 1.5. The finite dimensional $k G$-module $M$ is said to be of constant Jordan type if the Jordan type of $\alpha_{K}^{*}\left(M_{K}\right)$ is independent of the choice of $\pi$-point $\alpha_{K}: K[t] / t^{p} \longrightarrow K G$.

Remark 1.6. The Jordan type of $\alpha_{K}^{*}\left(M_{K}\right)$ for a finite dimensional $k G$-module $M$ at a $\pi$-point $\alpha_{K}: K[t] / t^{p} \longrightarrow K G$ typically depends not only upon the equivalence class $\left[\alpha_{K}\right] \in \Pi(G)$ but also upon the representative of this equivalence class. However, there are some exceptions. The central conclusion of [21] is that in either of the following two situations, the Jordan type of $\alpha_{K}^{*}\left(M_{K}\right)$ does not change if we replace $\alpha_{K}$ by some $\beta_{L}$ with $\alpha_{K} \sim \beta_{L}$ :

(1) If $\left[\alpha_{K}\right] \in \Pi(G)$ is a generic point; otherwise said, if $\alpha_{K}$ is a generic $\pi$-point.

(2) If for the given finite dimensional $k G$-module $M$, there does not exist any $\pi$-point $\beta_{L}$ such that the Jordan type of $\beta_{L}^{*}\left(M_{L}\right)$ is strictly greater than the Jordan type of $\alpha_{K}^{*}\left(M_{K}\right)$. In this situation we say that $\alpha_{K}$ has maximal Jordan type on $M$.

We recall [21, 5.1] that the non-maximal support variety, $\Gamma(G)_{M} \subset \Pi(G)$ associated to a finite dimensional $k G$-module $M$ is defined to be the (closed) subspace of those points $x \in \Pi(G)$ with the property that for some (and thus any) representative $\alpha_{K}$ of $x$ the Jordan type of $\alpha_{K}^{*}\left(M_{K}\right)$ is not maximal for $M$, or equivalently, $\alpha_{K}$ does not have maximal Jordan type on $M$. 
Remark 1.6(2) immediately leads us to the following equivalent formulation of the property of constant Jordan type.

Proposition 1.7. A finite dimensional $k G$-module is of constant Jordan type $a_{p}[p]+$ $\cdots+a_{1}[1]$ if and only if for each equivalence class $\left[\alpha_{K}\right] \in \Pi(G)$ there exists some representative $\alpha_{K}: K[t] / t^{p} \longrightarrow K G$ with the property $\alpha_{K}^{*}\left(M_{K}\right)$ has type $a_{p}[p]+$ $\cdots+a_{1}[1]$.

Since any $\pi$-point $\alpha_{K}: K[t] / t^{p} \longrightarrow K G$ has the property that $\alpha_{K}^{*}$ commutes with direct sums and (modulo projectives) Heller shifts, we conclude the following.

Proposition 1.8. Let $G$ be an arbitrary finite group scheme.

- The trivial $k G$-module $k$ has constant Jordan type.

- A finite dimensional projective $k G$-module has constant Jordan type. If $k G$ is not semi-simple, then the Jordan type of a $k G$-projective module $P$ is equal to $\frac{\operatorname{dim}_{k} P}{p}[p]$.

- If $\Omega^{i}(k)$ denotes the $i$-th Heller shift of $k$ for some $i \in \mathbb{Z}$, then $\Omega^{i}(k)$ has constant Jordan type equal to $n[p]+1[1]$ for some $n \geq 0$ if $i$ is even and equal to $m[p]+1[p-1]$ for some $m \geq 0$ if $i$ is odd.

- If $M$ has constant Jordan type, then $\Omega^{i}(M)$ also has constant Jordan type for any $i \in \mathbb{Z}$

- If $M, M^{\prime}$ are $k G$-modules of constant Jordan type, then $M \oplus M^{\prime}$ also has constant Jordan type.

The preceding proposition will be supplemented in subsequent sections by propositions asserting that other familiar operations on modules of constant Jordan type yield modules of constant Jordan type: taking a direct summand (by Theorem 3.7), taking the tensor product (by Corollary 4.3), and taking $\operatorname{Hom}_{k}(-,-)$ (by 5.4).

We make explicit the following elementary functoriality property.

Proposition 1.9. If $f: H \longrightarrow G$ is a flat map of finite group schemes and if $M$ is a $k G$-module of constant Jordan type, then $f^{*}(M)$ is a $k H$-module of the same constant Jordan type.

Proof. If $\alpha_{K}: K[t] / t^{p} \longrightarrow K H$ is a $\pi$-point of $H$, then $f \circ \alpha_{K}$ is a $\pi$-point of $G$ and the Jordan type of $\left.\alpha_{K}^{*}\left(\left(f^{*} M\right)\right)_{K}\right)$ equals that of $\left(f \circ \alpha_{K}\right)^{*}\left(M_{K}\right)$.

Remark 1.10. To verify whether or not a given finite dimensional $k G$-module $M$ has constant Jordan type it suffices to check that the Jordan type of $\alpha_{K}^{*}\left(M_{K}\right)$ does not vary as $\left[\alpha_{K}\right] \in \Pi(G)$ ranges over closed points of $\Pi(G)$. Thus, it suffices to consider $\alpha_{K}$ with $K / k$ finite. In particular, if $k$ is algebraically closed, it suffices to consider $k$-rational points of $\Pi(G)$. The collection of these points is denoted by $P(G)$, and was investigated extensively in [18]. 
Because a $k G$-module $M$ has constant Jordan type if and only if its base change $M_{K}$ has constant Jordan type as a $K G$-module for any field extension $K / k$, one could replace $k$ by its algebraic closure and consider only $k$-rational points for the algebraically closed field $k$.

\section{EXAMPles of MODUles of CONSTANT JORDAN TYPE}

We give examples of modules of constant Jordan type in special situations. Perhaps it is worth remarking that these examples are quite different from endotrivial modules considered in $\$ 5$,

Proposition 2.1. Let $E$ be an elementary abelian p-group and let $I \subset k E$ be the augmentation ideal of the group algebra $k E$. Then, for any $n \geq m, I^{m} / I^{n}$ is a $k E$-module of constant Jordan type.

Proof. Let $\beta: k[t] / t^{p} \longrightarrow k E \simeq k\left[t_{1}, \ldots, t_{r}\right] /\left(t_{1}^{p}, \ldots, t_{r}^{p}\right)$ be the $\pi$-point defined by $\beta(t)=t_{1}$ and let $\alpha_{K}: K[t] / t^{p} \longrightarrow K E$ be an arbitrary $\pi$-point. Since $\alpha_{K}$ is flat and $\alpha_{K}(t)$ has $p$-th power $0, \alpha_{K}(t)$ is a polynomial in $t_{1}, \ldots, t_{r}$ with constant term 0 and non-vanishing linear term $([18,2.2,2.6])$. Consequently, we may choose an automorphism $\theta_{\alpha}: K E \longrightarrow K E$ which sends $t_{1}$ to $\alpha_{K}(t)$, so that $\alpha_{K}=\theta_{\alpha} \circ \beta$. The automorphism $\theta_{\alpha}$ necessarily sends any power $I^{m}$ of the augmentation ideal isomorphically onto itself, so that

$$
\theta_{\alpha}^{-1}: \alpha_{K}^{*}\left(I^{m} / I^{n}\right)=\left(\theta_{\alpha} \circ \beta\right)^{*}\left(\left(I^{m} / I^{n}\right)_{K}\right) \simeq\left(\beta^{*}\left(I^{m} / I^{n}\right)\right)_{K}
$$

In other words, the Jordan type of $\alpha_{K}^{*}\left(I^{m} / I^{n}\right)$ does not depend upon the choice of $\pi$-point $\alpha_{K}$.

Example 2.2. As an elementary, specific case of Proposition 2.1, we consider $k E / I^{2}$ where $E$ is an elementary abelian $p$-group of rank $r$. This is a $k E$-module of dimension $r+1$ and can be represented explicitly as follows. Give the $k E$-module structure on $k^{r+1}$ by defining the generators $\left\{t_{1}, t_{2}, \ldots, t_{r}\right\}$ to act by multiplication by $\left\{e_{1,2}, e_{1,3}, \ldots, e_{1, r+1}\right\}$, pairwise commuting elementary matrices of size $(r+1) \times(r+1)$ with $p$-th power 0 . The constant Jordan type of the module $k E / I^{2}$ is $1[2]+(r-1)[1]$.

Remark 2.3. By Proposition 5.2, the dual $\left(k E / I^{2}\right)^{\#}$ of $k E / I^{2}$ is also a module of constant Jordan type of the same Jordan type as $k E / I^{2}$. In the special case of $r=2$, $\left(k E / I^{2},\left(k E / I^{2}\right)^{\#}\right)$ constitute the example produced years ago by Jens Jantzen of two non-isomorphic modules with the same "local Jordan type."

Example 2.4. We give a somewhat more interesting example to show how the residue characteristic $p$ plays a role. Consider the elementary abelian $p$-group $E$ of rank 2 , so that $k E=k[x, y] /\left(x^{p}, y^{p}\right)$. Define the $k E$-module $W$ of dimension 13 generated by $v_{1}, v_{2}, v_{3}, v_{4}$ and spanned as a $k$-vector space by

$$
\left\{v_{1}, v_{2}, v_{3}, v_{4}, x\left(v_{1}\right), x\left(v_{2}\right), x\left(v_{3}\right), x\left(v_{4}\right), x^{2}\left(v_{1}\right), x^{2}\left(v_{2}\right), x^{2}\left(v_{3}\right), y\left(v_{1}\right), y x\left(v_{1}\right)\right\}
$$


with

$$
x\left(v_{i}\right)=y\left(v_{i+1}\right), y^{2}\left(v_{1}\right)=x^{2}\left(v_{4}\right)=x^{3}\left(v_{i}\right)=0 .
$$

We represent this module with the following diagram

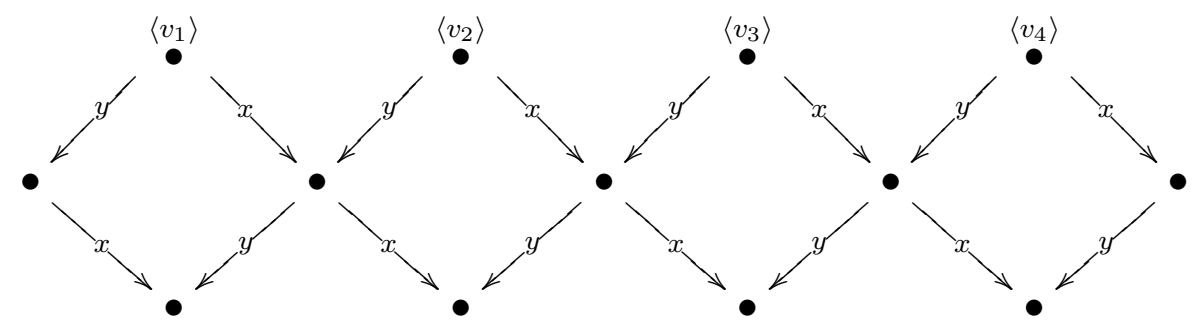

The vertices correspond to $k$-linear space generators, and the arrows indicate the action of the generators $x$ and $y$ of the group algebra. A vertex with no out-coming arrows corresponds to a trivial 1-dimensional submodule.

If $p=5$, then $W \simeq I^{3} / I^{6}$, and $W$ has constant Jordan type 3[3] $+2[2]$. More generally, the Jordan type of the $k E$-module $I^{p-2} / I^{p+1}$ is $(p-2)[3]+2[2]$.

If $p>5$, then $W$ does not have constant Jordan type. Namely, the Jordan type for both $x$ and $y$ on $W$ is $3[3]+2[2]$, whereas the Jordan type of $x+y$ is $4[3]+1[1]$. The Jordan blocks of size 3 for the action of $x+y$ are generated by $v_{1}, v_{2}, v_{3}$ and $v_{4}$ and the trivial block is generated by $x\left(v_{1}-v_{2}+v_{3}-v_{4}\right)+y\left(v_{4}\right)$.

Proposition 2.5. Let $s l_{2}$ denote the p-restricted Lie algebra of $2 \times 2$ matrices of trace 0 and let $u\left(s l_{2}\right)$ denote the restricted enveloping algebra of $s l_{2}$, the group algebra of the height 1 infinitesimal group scheme $G=S L_{2(1)}$. If the finite dimensional $u\left(s l_{2}\right)$ module $M$ is the restriction of a rational $S L_{2}$-module, then $M$ has constant Jordan type. On the other hand, other $u\left(s l_{2}\right)$-modules typically do not have constant Jordan type.

Proof. Let $\rho: S L_{2} \longrightarrow G L_{m}$ determine a rational $S L_{2}$-module $M$ of dimension $m$. Then the induced map of $p$-restricted Lie algebras $d \rho: s l_{2} \longrightarrow g l_{m}$ defines the associated $u\left(s l_{2}\right)$-module structure on $M$. For any field extension $K / k$ and any $x \in S L_{2}(K)$, the rational $S L_{2, K^{-}}$module $M_{K}^{\rho(x)}$ given by $A d(\rho(x)) \circ \rho$ is isomorphic to $M_{K}$ and thus the associated $u\left(s l_{2, K}\right)$-module $M_{K}^{\rho(x)}$ given by $d(A d(\rho(x) \circ \rho)$ is isomorphic to $M_{K}$.

Recall that the space of $k$-rational points of $\Pi(G)$ can be identified with the space of $k$-rational lines of the nilpotent variety $\mathcal{N}\left(s l_{2}\right)$. Indeed, each equivalence class of $\pi$-points is represented by some $\alpha_{K}: K[t] / t^{p} \longrightarrow u\left(s l_{2, K}\right)$ sending $t$ to a nilpotent matrix of $s l_{2}(K)$. Moreover, the Jordan type of a given finite dimensional $u\left(s l_{2}\right)$ module does not depend upon the choice of such a representative of a given point of $\Pi(G)$ by [21, 3.1]. The action of $S L_{2}(K)$ on $\Pi(G)_{K}$ sending $\alpha_{K}$ to $d \rho(A d(x)) \circ \alpha_{K}=$ $A d(\rho(x)) \circ \alpha_{K}$ corresponds to the natural action of $S L_{2}$ on $\mathcal{N}\left(s l_{2}\right)$. Consequently, 
the transitivity of this action together with Proposition 1.7 implies that the rational $S L_{2}$-module $M$ has constant Jordan type as a $u\left(s l_{2}\right)$-module.

On the other hand, any $u\left(s l_{2}\right)$-module $M$ whose $\Pi$-support space $\Pi(G)_{M}$ is a non-empty proper subset of the 1-dimensional variety $\Pi(G)$ has Jordan type of $M$ at a generic $\pi$-point of $\Pi(G)$ equal to $\frac{m}{p}[p]$ (where $m=\operatorname{dim} M$ ) and strictly smaller Jordan type at any $\pi$-point representing a point in $\Pi(G)_{M}$. Such $M$ abound, since every finite subset of $\Pi(G)$ is of the form $\Pi(G)_{M}$ for some finite dimensional $k G$ module $M$. For example, let $b \subset s l_{2}$ be the Lie subalgebra of lower triangular matrices and consider the $u\left(s l_{2}\right)$-module $M=u\left(s l_{2}\right) \otimes_{u(b)} k$, the module obtained from the trivial $u(b)$-module by coinduction. Then $M$ is free when restricted to the 1-dimensional subalgebra of strictly upper triangular matrices and trivial when restricted to the 1-dimensional subalgebra of strictly lower triangular matrices.

We conclude this section of explicit examples with a family of modules $V_{n}, n>0$ which are modules of constant Jordan type $n[2]+1[1]$ regardless of the prime $p$. The module $V_{n}$ can be represented by the following diagram:
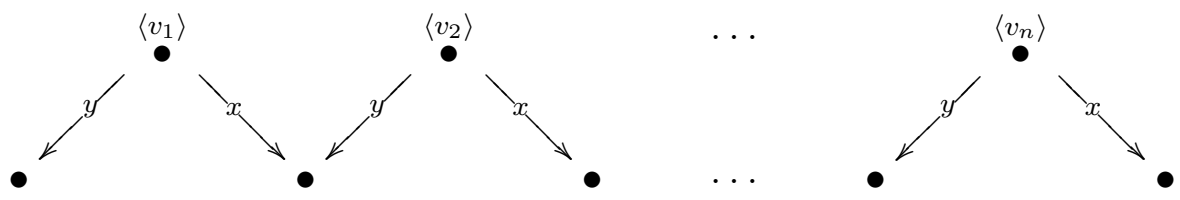

The verification of the assertion that $V_{n}$ does indeed have constant Jordan type is a simple computation.

In the following proposition we describe the modules $V_{n}$ explicitly in terms of generators and relations.

Proposition 2.6. Consider a rank 2 elementary abelian p-group $E$, so that $k E=$ $k[x, y] /\left(x^{p}, y^{p}\right)$. Consider the $k E$-module $V_{n}$ of dimension $2 n+1$ generated by $v_{1}, \ldots, v_{n}$ spanned as a $k$-vector space by $\left\{v_{1}, \ldots, v_{n}, x\left(v_{1}\right), \ldots, x\left(v_{n}\right), y\left(v_{1}\right)\right\}$ with $x\left(v_{i}\right)=y\left(v_{i+1}\right), x^{2}\left(v_{i}\right)=x y\left(v_{i}\right)=y^{2}\left(v_{i}\right)=0$. Then $V_{n}$ has constant Jordan type $n[2]+1[1]$.

\section{Specialization, $\Gamma(G)_{M}$, And COnstant JoRdan type}

In this section, we introduce a strict form of specialization of $\pi$-points which was considered briefly in [21, 3.2] for infinitesimal group schemes. We also recall the non-maximal support variety $\Gamma(G)_{M}$ of a finite dimensional $k G$-module $M$. We use both strict specialization and the non-maximal support variety to further explore the class of modules of constant Jordan type.

The condition recalled in Definition 1.2 that the $\pi$-point $\alpha_{K}$ specializes to the $\pi$ point $\beta_{L}$ is equivalent to the algebro-geometric condition that the point $\left[\alpha_{K}\right] \in \Pi(G)$ specializes to $\left[\beta_{L}\right]([20])$. For some purposes, this notion of specialization is not sufficiently strong. Namely, if $\Pi(G)$ is reducible, then there exist finite dimensional 
$k G$-modules $M$ and $\pi$-points $\alpha_{K}, \beta_{L}$ such that $\alpha_{K}$ specializes to $\beta_{L}$ but the Jordan type of $\alpha_{K}^{*}\left(M_{K}\right)$ is smaller than the Jordan type of $\beta_{L}^{*}\left(M_{L}\right)$ (see [20,4.14]).

The following definition introduces a stricter definition of specialization which is a natural extension of [21, 3.2] from infinitesimal group schemes to arbitrary finite group schemes.

Definition 3.1. Let $G$ be a finite group scheme and $\alpha_{K}, \beta_{L}$ be $\pi$-points of $G$. We say that $\alpha_{K}$ strictly specializes to $\beta_{L}$ (and write $\alpha_{K} \downarrow \beta_{L}$ ), if there exists a commutative local domain $R$ over $k$ with field of fractions $K$ and residue field $L$, together with a map of $R$-algebras $\nu_{R}: R[t] / t^{p} \longrightarrow R G$ such that $\nu_{R} \otimes_{R} K=\alpha_{K}, \nu_{R} \otimes_{R} L=\beta_{L}$.

Theorem 3.2. Let $R$ be a commutative local domain with field of fractions $K$ and residue field $L$. Let $A \in M_{N}(R)$ be an $N \times N$ matrix which has $p$-th power 0 and coefficients in $R$. Then the Jordan type of $A \otimes_{R} K \in M_{N}(K)$ is greater or equal to the Jordan type of $A \otimes_{R} L \in M_{N}(L)$.

Consequently, if $\alpha_{K}, \beta_{L}$ are $\pi$-points of a finite group scheme $G$ with $\alpha_{K} \downarrow \beta_{L}$ and if $M$ is a finite dimensional $k G$-module, then $\alpha_{K}^{*}\left(M_{K}\right)$ has Jordan type greater or equal to that of $\beta_{L}^{*}\left(M_{L}\right)$. In particular, $\alpha_{K} \downarrow \beta_{L}$ implies $\alpha_{K} \downarrow \beta_{L}$.

Proof. Let $M[i]$ denote the cokernel of $A^{i}: R^{N} \longrightarrow R^{N}$ for some $i<p$. Let $m_{1}, \ldots, m_{s} \in M[i]$ be chosen so that $\bar{m}_{1}, \ldots, \bar{m}_{s} \in M[i] \otimes_{R} L$ is a basis, where $\bar{m}_{i}=m_{i} \otimes 1_{L}$. By Nakayama's Lemma, $m_{1}, \ldots, m_{s}$ generate $M[i]$ as an $R$-module and thus their images span $M[i] \otimes_{R} K$. Observe that $M[i] \otimes_{R} K$ is the cokernel of $\left(A \otimes_{R} K\right)^{i}: K^{N} \longrightarrow K^{N}$ and that $M[i] \otimes_{R} L$ is the cokernel of the homomorphism $\left(A \otimes_{R} L\right)^{i}: L^{N} \longrightarrow L^{N}$. This implies that the rank of $\left(A \otimes_{R} K\right)^{i}$ is greater or equal to the rank of $\left(A \otimes_{R} L\right)^{i}$ for any $i<p$ so that the Jordan type of $A \otimes_{R} K \in M_{N}(K)$ is greater or equal to the Jordan type of $A \otimes_{R} L \in M_{N}(L)$.

The second statement is a special case of the first. The last statement follows from the observation that if $\beta_{L}^{*}\left(M_{L}\right)$ is projective then its Jordan type is the maximal possible Jordan type on $M$ and hence $\alpha_{K}^{*}\left(M_{K}\right)$ must also be projective assuming that $\alpha_{K} \downarrow \beta_{L}$.

The next theorem verifies that specialization of points in $\Pi(G)$ can be represented by strict specialization.

Theorem 3.3. Let $G$ be a finite group scheme and let $\alpha_{K}, \beta_{L}$ be $\pi$-points of $G$ with $\alpha_{K} \downarrow \beta_{L}$. Then there exist $\pi$-points $\alpha_{K^{\prime}}^{\prime} \sim \alpha_{K}$ and $\beta_{L^{\prime}}^{\prime} \sim \beta_{L}$ such that $\alpha_{K^{\prime}}^{\prime} \downarrow \beta_{L^{\prime}}^{\prime}$.

Proof. Using [20, 4.13], we can choose an elementary abelian $p$-group $E \subset \pi_{0}(G)$ such that $\left[\alpha_{K}\right],\left[\beta_{L}\right]$ are in the image of the closed map $\Pi\left(\left(G^{0}\right)^{E} \times E\right) \longrightarrow \Pi(G)$. Observe that the group algebra of $\left(G^{0}\right)^{E} \times E$ is isomorphic to the group algebra of some infinitesimal group scheme $H$. Because the relationship of strict specialization given in Definition 3.1 and the definition of specialization given in Definition 1.2 
involve only the group algebra of the given finite group scheme, we may replace $G$ by $H$. Thus, we assume $G$ is an infinitesimal group scheme.

Let Spec $A \subset \Pi(G)$ be an irreducible affine open subset containing $\left[\beta_{L}\right]$. Since $\alpha_{K} \downarrow \beta_{L}$, we have that $\left[\beta_{L}\right]$ is in the closure of $\left[\alpha_{K}\right]$ by $[20,4.3]$. Hence, any closed irreducible component in the complement of Spec $A$ containing $\left[\alpha_{K}\right]$ must contain $\left[\beta_{L}\right]$. We conclude that $\left[\alpha_{K}\right] \in \operatorname{Spec} A$.

Replacing $A$ by $A_{\text {red }}$, we may assume that $A$ is a domain. Let $R$ denote the local $A$-algebra defined as the localization at the prime corresponding to $\left[\alpha_{K}\right]$ of the quotient of $A$ by the prime corresponding to $\left[\beta_{L}\right]$. Set $K^{\prime}$ to be the field of fractions of $R$ and $L^{\prime}$ to be the residue field of $R$.

Let $r$ denote the height of the infinitesimal group scheme $G$ and let $V_{r}(G)$ be the scheme of 1-parameter subgroups of $G$. Recall that the natural morphism $\Theta_{G}: V_{r}(G) \backslash\{0\} \longrightarrow \Pi(G)$, which is determined by sending a 1-parameter subgroup $\mu: \mathbb{G}_{a(r), K} \longrightarrow G_{K}$ to the $\pi$-point $\mu_{*} \circ \epsilon: K[t] / t^{p} \longrightarrow K \mathbb{G}_{a(r)} \longrightarrow K G$, where $\epsilon$ has the property that its composition with the map on group algebras induced by the projection $\mathbb{G}_{a(r)} \longrightarrow \mathbb{G}_{a(r)} / \mathbb{G}_{a(r-1)}$, is an isomorphism. Because $\Theta_{G}$ together with the isogeny $\mathrm{H}^{\bullet}(G, k) \rightarrow k\left[V_{r}(G)\right]$ of [26, 5.2] induces the isomorphism $\operatorname{Proj}\left(V_{r}(G)\right) \longrightarrow \Pi(G)$ of Theorem 1.4, we conclude that the given morphism Spec $R \longrightarrow \Pi(G)$ lifts to a morphism

$$
\operatorname{Spec} R \longrightarrow\left(\operatorname{Spec} H^{\bullet}(G, k)[1 / P]\right)_{0} \subset V_{r}(G),
$$

which corresponds to a morphism of $R$-group schemes $\nu: \mathbb{G}_{a(r), R} \longrightarrow G_{R}$. Here, $P$ is a homogeneous element of positive degree of $\mathrm{H}^{\bullet}(G, k)$ which does not vanish on the image of the closed point of Spec $R$ and $\left(A^{\bullet}\right)_{0}$ denotes the subalgebra of degree 0 elements of the graded algebra $A^{\bullet}$.

We define $\nu_{R}$ to be the map of $R$-algebras given as the composition

$$
R[t] / t^{p} \stackrel{\epsilon}{\longrightarrow} R\left(\mathbb{G}_{a(r), R}\right) \stackrel{\nu_{*}}{\longrightarrow} R G .
$$

By construction, $\nu_{R} \otimes_{R} K^{\prime}$ is a $\pi$-point of $G$ in the equivalence class $\left[\alpha_{K}\right]$ (the image of Spec $K^{\prime}$ ) and $\nu_{R} \otimes_{R} L^{\prime}$ is a $\pi$-point of $G$ in the equivalence class $\left[\beta_{L}\right]$.

The proof of the theorem in the title of [8] applies with only minor notational change to prove the same assertion, given below, for an arbitrary finite group scheme.

Theorem 3.4. ([8]) Let $G$ be a finite group scheme and $M$ a finite dimensional indecomposable $k G$-module. Then $\Pi(G)_{M}$ is connected. In particular, $\Pi(G)=\Pi(G)_{k}$ is connected.

Theorems 3.3 and 3.4 enable the following equivalent formulation of the condition that a module has constant Jordan type. 
Proposition 3.5. Let $G$ be a finite group scheme and let $M$ be a $k G$-module. Then $M$ has constant Jordan type if and only if $\alpha_{K}$ and $\beta_{L}$ have the same Jordan type on $M$ whenever $\alpha_{K}, \beta_{L}$ are $\pi$-points of $G$ satisfying $\alpha_{K} \downarrow \beta_{L}$.

Proof. If $M$ has constant Jordan type and $\alpha_{K}, \beta_{L}$ are $\pi$-points of $G$, then the Jordan types of $\alpha_{K}$ and and $\beta_{L}$ on $M$ are necessarily equal.

To prove the converse, we recall that if $x \in \Pi(G)$ is a point such that $M$ has maximal Jordan type at some representative of $x$, then the Jordan type on $M$ is the same for every representative of $x$ (see Remark 1.6). Let $x \in \Pi(G)$ be a point which has maximal Jordan type on $M$ and let $y \in \Pi(G)$ be an arbitrary point. Since $\Pi(G)$ is connected (by Theorem 3.4) and Noetherian, we may find a chain of irreducible components $Z_{0}, \ldots, Z_{r}$ of $\Pi(G)$ such that $Z_{i} \cap Z_{i+1} \neq \emptyset$ and $x \in Z_{0}, y \in Z_{r}$. Let $\eta_{i} \in Z_{i}$ be the generic point and choose some $z_{i} \in Z_{i} \cap Z_{i+1}$. Then $x, \eta_{0}, z_{1}, \eta_{1}, \ldots, z_{i}, \eta_{i}, \ldots, \eta_{r}, y$ is a chain of points $x=x_{0}, x_{1}, \ldots, x_{n}=y \in \Pi(G)$ such that there exist morphisms $\operatorname{Spec} R_{i} \longrightarrow \Pi(G)_{\text {red }}$ sending $\left\{\operatorname{Spec} K_{i}, \operatorname{Spec} L_{i}\right\}$ to the (unordered) pair $\left\{x_{i-1}, x_{i}\right\}$ where $R_{i}$ is a commutative local domain with field of fractions $K_{i}$ and residue field $L_{i}$.

Our hypothesis in conjunction with Theorem 3.3 implies that there are representing $\pi$-points $\alpha_{K_{i}}, \alpha_{L_{i}}$ of $x_{i}$ and $x_{i-1}$ at which $M$ has the same Jordan type. Consequently, $M$ has the same maximal Jordan type at each representative of each $x_{i}$. Thus, the Jordan type of $M$ at any representative of $x_{n}=y$ equals this same maximal Jordan type; in other words, $M$ has constant Jordan type.

We give a useful characterization of modules $M$ of constant Jordan type in terms of their non-maximal support varieties $\Gamma(G)_{M}$ recalled in Remark 1.6.

Proposition 3.6. Let $M$ be a finite dimensional $k G$-module. Then $M$ has constant Jordan type if and only if $\Gamma(G)_{M}=\emptyset$.

Proof. If $M$ has constant Jordan type, then clearly $\Gamma(G)_{M}=\emptyset$. Conversely, let $\Gamma(G)_{M}=\emptyset$, and let $\alpha_{K}, \beta_{L}$ be any two $\pi$-points of $G$ satisfying $\alpha_{K} \downarrow \beta_{L}$. By Theorem $3.2, \alpha_{K}^{*}\left(M_{K}\right)$ has Jordan type greater or equal to that of $\beta_{L}^{*}\left(M_{L}\right)$. Since $\Gamma(G)_{M}=\emptyset$, we must have that the Jordan types of $\alpha_{K}^{*}\left(M_{K}\right)$ and $\beta_{L}^{*}\left(M_{L}\right)$ are the same. Hence, Proposition 3.5 implies that $M$ has constant Jordan type.

The following "closure property" of modules of constant Jordan type is somewhat striking.

Theorem 3.7. Let $M$ be a $k G$-module of constant Jordan type. Then any direct summand of $M$ also has constant Jordan type.

Proof. Write $M=M^{\prime} \oplus M^{\prime \prime}$. Let $\alpha_{K}, \beta_{L}$ be two $\pi$-points such that $\alpha_{K} \downarrow \beta_{L}$. By Theorem 3.2, we have

$$
\operatorname{JType}\left(\alpha_{K}^{*}\left(M_{K}^{\prime}\right)\right) \geq \operatorname{JType}\left(\beta_{L}^{*}\left(M_{L}^{\prime}\right)\right) \text { and } \operatorname{JType}\left(\alpha_{K}^{*}\left(M_{K}^{\prime \prime}\right)\right) \geq \operatorname{JType}\left(\beta_{L}^{*}\left(M_{L}^{\prime \prime}\right)\right) \text {. }
$$


Hence,

$$
\begin{aligned}
\operatorname{JType}\left(\alpha_{K}^{*}\left(M_{K}^{\prime}\right)\right) \oplus & \operatorname{JType}\left(\alpha_{K}^{*}\left(M_{K}^{\prime \prime}\right)\right)=\operatorname{JType}\left(\alpha_{K}^{*}(M)\right)=\operatorname{JType}\left(\beta_{L}^{*}\left(M_{L}\right)\right)= \\
& =\operatorname{JType}\left(\beta_{L}^{*}\left(M_{L}^{\prime}\right)\right) \oplus \operatorname{JType}\left(\beta_{L}^{*}\left(M_{L}^{\prime \prime}\right)\right),
\end{aligned}
$$

where JType $(-)$ is the Jordan type function. Therefore, both inequalities in (3) must be equalities. Since this holds for any pair $\alpha_{K} \downarrow \beta_{L}$, the statement now follows immediately from Proposition 3.5.

\section{Behavior With Respect to Tensor PRODUCtS}

We begin with the following "order preserving" property of Jordan types. The proof given below uses an explicit description of the tensor product of indecomposable $k[t] / t^{p}$-modules which is presented in the Appendix. The coalgebra structure we (implicitly) give $k[t] / t^{p}$ is that for which $t$ is primitive (i.e., $\nabla(t)=t \otimes 1+1 \otimes t$ ).

Proposition 4.1. Let $M, N$ and $L$ be $k[t] / t^{p}$-modules with Jordan types $\underline{a}=a_{p}[p]+$ $\cdots+a_{1}[1], \underline{b}=b_{p}[p]+\cdots+b_{1}[1]$ and $\underline{c}=c_{p}[p]+\cdots+c_{1}[1]$ respectively such that $\operatorname{dim} M=\operatorname{dim} N$. Let $\underline{a} \otimes \underline{c}, \underline{b} \otimes \underline{c}$ denote the Jordan types of $M \otimes L, N \otimes L$ respectively.

If $\underline{a} \geq \underline{b}$, then $\underline{a} \otimes \underline{c} \geq \underline{b} \otimes \underline{c}$.

If $\underline{a}>\underline{b}$ and if $c_{i} \neq 0$ for some $i<p$, then $\underline{a} \otimes \underline{c}>\underline{b} \otimes \underline{c}$.

Proof. For a $k[t] / t^{p}$-module $M$ of dimension $m$, we denote the representation afforded by $M$ by $\rho_{M}: k[t] / t^{p} \longrightarrow \operatorname{End}_{k}(A) \simeq M_{m}(k)$. The dominance condition (1) on Jordan types $\underline{a} \geq \underline{b}$ of $M$ and $N$ both of dimension $m$ can be formulated as the condition that

$$
\operatorname{Rk} \rho_{M}(t)^{i} \geq \operatorname{Rk} \rho_{N}(t)^{i}, \quad 1 \leq i<p
$$

or equivalently that

$$
\operatorname{dim} \operatorname{Ker}\left\{\rho_{M}(t)^{i}\right\} \leq \operatorname{dim} \operatorname{Ker}\left\{\rho_{N}(t)^{i}\right\}, \quad 1 \leq i<p .
$$

For $\underline{a}>\underline{b}$, the additional condition is that $\operatorname{Rk} \rho_{M}(t)^{i}>\operatorname{Rk} \rho_{N}(t)^{i}$ for some $i$.

If $L \simeq L_{1} \oplus L_{2}$, so that $\underline{c}=\underline{c}_{1}+\underline{c}_{2}$, then we immediately conclude that

$$
\underline{a} \otimes \underline{c}=\underline{a} \otimes \underline{c}_{1}+\underline{a} \otimes \underline{c}_{2} .
$$

Thus, we may assume that $L$ is indecomposable, say $L=[\ell]$.

Denote by $J_{s} \in M_{s}(k)$ the Jordan block of size $s$. Then $J_{s}=\rho_{[s]}(t)$ once we choose an appropriate basis. Since $t$ acts on $M \otimes[\ell]$ as $t \otimes 1+1 \otimes t$, we get the formula

$$
\rho_{M \otimes[\ell]}(t)=\rho_{M}(t) \otimes \operatorname{Id}_{[s]}+\operatorname{Id}_{M} \otimes J_{s}
$$

Lemma 1.10 of [21] implies

$$
\operatorname{Ker}\left\{\rho_{M \otimes[\ell]}(t)\right\} \cong \operatorname{Ker}\left\{\rho_{M}(t)^{\ell}\right\}
$$


Applying the same argument to $M \otimes[\ell]$, we obtain

$$
\operatorname{Ker}\left\{\rho_{M \otimes[\ell]}(t)^{s}\right\}=\operatorname{Ker}\left\{\rho_{M \otimes[\ell] \otimes[s]}(t)\right\}
$$

We write $[\ell] \otimes[s]=\bigoplus_{j} C_{\ell s}^{j}[j]$ where the coefficients $C_{\ell s}^{j}$ are given by the Corollary 10.2 of the Appendix. With this notation,

$$
\begin{gathered}
\operatorname{dim} \operatorname{Ker}\left\{\rho_{M \otimes[\ell]}(t)^{s}\right\}=\operatorname{dim} \operatorname{Ker}\left\{\rho_{M \otimes\left(\oplus C_{\ell s}^{j}[j]\right)}(t)\right\}=\operatorname{dim} \operatorname{Ker}\left\{\rho_{\oplus C_{\ell s}^{j}(M \otimes[j])}(t)\right\} \\
=\sum C_{\ell s}^{j} \operatorname{dim} \operatorname{Ker}\left\{\rho_{M \otimes[j]}(t)\right\}=\sum C_{\ell s}^{j} \operatorname{dim} \operatorname{Ker}\left\{\rho_{M}(t)^{j}\right\} .
\end{gathered}
$$

Consequently,

$$
\operatorname{dim} \operatorname{Ker}\left\{\rho_{M \otimes[\ell]}(t)^{s}\right\}=\sum C_{\ell s}^{j} \operatorname{dim} \operatorname{Ker}\left\{\rho_{M}(t)^{j}\right\}
$$

and

$$
\operatorname{dim} \operatorname{Ker}\left\{\rho_{N \otimes[\ell]}(t)^{s}\right\}=\sum C_{\ell s}^{j} \operatorname{dim} \operatorname{Ker}\left\{\rho_{N}(t)^{j}\right\}
$$

Hence, (4) implies that

$$
\operatorname{dim} \operatorname{Ker}\left\{\rho_{M \otimes[\ell]}(t)^{s}\right\} \leq \operatorname{dim} \operatorname{Ker}\left\{\rho_{N \otimes[\ell]}(t)^{s}\right\}
$$

for all $s$. Therefore, $\underline{a} \otimes \underline{c} \geq \underline{b} \otimes \underline{c}$.

Now assume $\underline{a}>\underline{b}$ and that some $c_{i} \neq 0, i<p$. Then, we may assume that $L=[\ell]$ with $\ell<p$. Choose $j$ such that $\operatorname{dim} \operatorname{Ker}\left\{\rho_{M}(t)^{j}\right\}<\operatorname{dim} \operatorname{Ker}\left\{\rho_{N}(t)^{j}\right\}$. The formula of Corollary 4.3 implies that there exists some $s$ such that $C_{\ell s}^{j}=1$. That is, if $j \geq \ell$, then take $s=j-\ell+1$, and if $j<\ell$, take $s=\ell-j+1$. For such $s$ we get the strict inequality

$$
\operatorname{dim} \operatorname{Ker}\left\{\rho_{M \otimes[\ell]}(t)^{s}\right\}<\operatorname{dim} \operatorname{Ker}\left\{\rho_{N \otimes[\ell]}(t)^{s}\right\}
$$

Therefore, $\underline{a} \otimes \underline{c}>\underline{b} \otimes \underline{c}$.

Proposition 4.1 enables us to establish various tensor product properties of maximal Jordan type. We should point out that there are some subtleties which must be carefully considered. For example if $M$ and $N$ are $k G$-modules and $\alpha_{K}$ is a $\pi$-point whose image is not a sub-Hopf algebra of $K G$, then it is not always true that $\alpha_{K}^{*}\left(M_{K} \otimes N_{K}\right) \simeq \alpha_{K}^{*}\left(M_{K}\right) \otimes \alpha_{K}^{*}\left(N_{K}\right)$. Hence, Proposition 4.1 can not be applied directly. The somewhat surprising Examples 4.5 and 4.6 indicate the subtle relationship between tensor products and maximal Jordan types. In view of these examples, we take some care in the proofs of the following properties.

Theorem 4.2. Let $G$ be a finite group scheme, and consider two finite dimensional $k G$-modules $M, N$ and a $\pi$-point $\alpha_{K}$ of $G$.

(1) If $\alpha_{K}$ has maximal Jordan type on both $M$ and $N$, then

$$
\alpha_{K}^{*}\left(M_{K} \otimes N_{K}\right) \simeq \alpha_{K}^{*}\left(M_{K}\right) \otimes \alpha_{K}^{*}\left(N_{K}\right) .
$$


(2) If $\alpha_{K}$ has maximal Jordan type on $M \otimes N$, then

$$
\alpha_{K}^{*}\left(M_{K} \otimes N_{K}\right) \simeq \alpha_{K}^{*}\left(M_{K}\right) \otimes \alpha_{K}^{*}\left(N_{K}\right),
$$

(3) If $\Pi(G)$ is irreducible and if $\alpha_{K}$ has maximal Jordan type on both $M$ and $N$, then $\alpha_{K}$ has maximal Jordan type on $M \otimes N$ and that Jordan type is equal to the Jordan type of $\alpha_{K}^{*}\left(M_{K}\right) \otimes \alpha_{K}^{*}\left(N_{K}\right)$.

Proof. Let $i: C_{K} \subset G_{K}$ be a unipotent abelian subgroup scheme through which $\alpha_{K}$ factors. Since $i: K C_{K} \longrightarrow K G_{K}$ is a map of Hopf algebras, $i^{*}$ commutes with tensor products. Observe that the maximality of $\alpha_{K}$ on $M, N$ or $M \otimes N$ as $k G$ modules implies the maximality of $\alpha_{K}$ on $M_{K}, N_{K}$ or $M_{K} \otimes_{K} N_{K}$ as $K C_{K}$-modules. Because $\Pi\left(C_{K}\right)$ is irreducible, the maximal Jordan types of $M_{K}, N_{K}$, and $M_{K} \otimes_{K} N_{K}$ are all achieved at a generic $\pi$-point of $\Pi\left(C_{K}\right)$. Thus, statement (1) follows from [21, 4.4] applied to $C_{K}$.

To prove (2), we extend scalars if necessary so that $K$ is perfect. Hence, $K C_{K} \simeq$ $K\left[T_{1}, \ldots, T_{r}\right] /\left(T_{1}^{p^{e_{1}}}, \ldots, T_{r}^{p^{e_{r}}}\right)($ see $[28,14.4])$. Let $t_{i}=T_{i}^{p^{e_{i}-1}}$.

Let $\rho_{M}: K C_{K} \longrightarrow \operatorname{End}_{K}\left(M_{K}\right)$ be the map defined by the representation $M_{K}$ of $C_{K}$, and similarly define $\rho_{N}$ and $\rho_{M \otimes N}$. Choose a generic $\pi$-point $\eta_{\Omega}$ of $C_{K}$, $\eta_{\Omega}: \Omega[t] / t^{p} \longrightarrow \Omega C_{K}$, where $\Omega / K$ is a field extension. Arguing exactly as in the proof of [21, 4.4], we conclude that the Jordan type of $\rho_{M}\left(\eta_{\Omega}(t)\right) \otimes 1+1 \otimes \rho_{N}\left(\eta_{\Omega}(t)\right)$ as an element of $\operatorname{End}_{\Omega}\left(M_{\Omega} \otimes N_{\Omega}\right)$ is greater or equal than the Jordan type of any linear combination of elements of the form $\rho_{M}\left(t_{i}\right) \otimes 1$ and $1 \otimes \rho_{N}\left(t_{j}\right), 1 \leq i, j \leq r$. Since $\eta_{\Omega}^{*}\left(M_{\Omega} \otimes N_{\Omega}\right) \simeq \eta_{\Omega}^{*}\left(M_{\Omega}\right) \otimes \eta_{\Omega}^{*}\left(N_{\Omega}\right)$ by [21, 4.4], the same conclusion holds for $\rho_{M \otimes N}\left(\eta_{\Omega}(t)\right)$. Since $\alpha_{K}$ is maximal on $M \otimes N$ and $\eta_{\Omega}$ is a generic $\pi$-point, the Jordan type of $\alpha_{K}^{*}\left(M_{K} \otimes N_{K}\right)$ equals the Jordan type of $\eta_{\Omega}^{*}\left(M_{\Omega} \otimes N_{\Omega}\right)$. Hence, the Jordan type of $\rho_{M \otimes N}\left(\alpha_{K}(t)\right)$ is greater or equal than the Jordan type of any linear combination of elements of the form $\rho_{M}\left(t_{i}\right) \otimes 1$ or $1 \otimes \rho_{N}\left(t_{j}\right), 1 \leq i, j \leq r$.

Let $\nabla: K C_{K} \rightarrow K C_{K} \otimes K C_{K}$ be the coproduct on $K C_{K}$, and let $I=\left(T_{1}, \ldots, T_{r}\right)$ be the augmentation ideal of $K C_{K}$. and let $I^{(p)}$ be the ideal generated by $\left(t_{1}, \ldots, t_{r}\right)$. Recall that

$$
\alpha_{K}(t) \otimes 1+1 \otimes \alpha_{K}(t)-\nabla\left(\alpha_{K}(t)\right) \in I \otimes I
$$

(see [23, I.2.4]). Since $\alpha_{K}(t)$ has $p$-th power 0, we can refine this further, concluding that

$$
\alpha_{K}(t) \otimes 1+1 \otimes \alpha_{K}(t)-\nabla\left(\alpha_{K}(t)\right) \in I \otimes I^{(p)}+I^{(p)} \otimes I .
$$

The action of $K C_{K}$ on the tensor product $M_{K} \otimes N_{K}$ is given by the formula

$$
\rho_{M \otimes N}=\left(\rho_{M} \otimes \rho_{N}\right) \circ \nabla
$$

Hence,

$$
\begin{gathered}
\rho_{M}\left(\alpha_{K}(t)\right) \otimes 1+1 \otimes \rho_{N}\left(\alpha_{K}(t)\right)= \\
\rho_{M \otimes N}\left(\alpha_{K}(t)\right)+\left\langle\text { multiples of } \rho_{M}\left(t_{i}\right) \otimes 1 \text { and } 1 \otimes \rho_{N}\left(t_{j}\right)\right\rangle
\end{gathered}
$$


Since the Jordan type of $\rho_{M \otimes N}\left(\alpha_{K}(t)\right)$ is greater of equal than the Jordan type of any linear combination of elements $\rho_{M}\left(t_{i}\right) \otimes 1$ and $1 \otimes \rho_{N}\left(t_{j}\right)$ for $1 \leq i, j \leq r$, the relation (8) and Theorem 1.12 of [21] imply that $\rho_{M \otimes N}\left(\alpha_{K}(t)\right)$ and $\rho_{M}\left(\alpha_{K}(t)\right) \otimes 1+$ $1 \otimes \rho_{N}\left(\alpha_{K}(t)\right)$ have the same Jordan type. Hence, $\alpha_{K}^{*}(M) \otimes_{K} \alpha_{K}^{*}(N) \simeq \alpha_{K}^{*}\left(M_{K} \otimes_{K}\right.$ $\left.N_{K}\right)$.

To prove (3) we assume that $\Pi(G)$ is irreducible. Let $\eta_{\Omega}: \Omega[t] / t^{p} \longrightarrow \Omega G$ be a generic $\pi$-point of $G$. Applying [21, 4.4] to $\eta_{\Omega}$, we get

$$
\eta_{\Omega}^{*}\left(M_{\Omega}\right) \otimes_{\Omega} \eta_{\Omega}^{*}\left(N_{\Omega}\right) \simeq \eta_{\Omega}^{*}\left(M_{\Omega} \otimes_{\Omega} N_{\Omega}\right)
$$

Since $\Pi(G)$ is irreducible, the absolute maximal Jordan type of any finite dimensional $k G$-module is realized at $\eta_{\Omega}$. Hence, the maximality assumption on $\alpha_{K}$ on $M$ and $N$ implies that $\alpha_{K}^{*}\left(M_{K}\right)$ has the same Jordan type as $\eta_{\Omega}^{*}\left(M_{\Omega}\right)$ and $\alpha_{K}^{*}\left(N_{K}\right)$ has the same Jordan type as $\eta_{\Omega}^{*}\left(N_{\Omega}\right)$. We conclude that

$$
\alpha_{K}^{*}\left(M_{K} \otimes N_{K}\right) \simeq \alpha_{K}^{*}\left(M_{K}\right) \otimes \alpha_{K}^{*}\left(N_{K}\right)
$$

has the same Jordan type as

$$
\eta_{\Omega}^{*}\left(M_{\Omega}\right) \otimes_{\Omega} \eta_{\Omega}^{*}\left(N_{\Omega}\right) \simeq \eta_{\Omega}^{*}\left(M_{\Omega} \otimes_{\Omega} N_{\Omega}\right)
$$

where the first isomorphism follows from statement (1). Hence, the Jordan type of $\alpha_{K}$ on $M \otimes N$ is maximal.

An easy corollary of Theorem 4.2 is the following assertion that the tensor product of modules of constant Jordan type is again of constant Jordan type.

Corollary 4.3. Let $G$ be a finite group scheme and let $M, N$ be finite dimensional $k G$-modules. If $M$ and $N$ have constant Jordan type, then $M \otimes N$ also has constant Jordan type.

Proof. We merely observe that $\alpha_{K}$ has maximal Jordan type on both $M$ and $N$ for any $\pi$-point $\alpha_{K}: K[t] / t^{p} \longrightarrow K G$ whenever $M, N$ are of constant Jordan type. Thus, the corollary follows from the first statement of Theorem 4.2 .

The following consequence of Theorem 4.2 will be used to prove Proposition 4.7 .

Corollary 4.4. Let $G$ be a finite group scheme such that $\Pi(G)$ is irreducible and let $\alpha_{K}: K[t] / t^{p} \longrightarrow K G$ be a $\pi$-point of $G$. Let $M$ be a $k G$-module such that $\alpha_{K}^{*}\left(M_{K}\right)$ is not projective and let $N$ be another $k G$-module such that $\alpha_{K}$ does not have maximal Jordan type on $N$. Then $\alpha_{K}$ does not have maximal Jordan type on $M \otimes N$.

Proof. If the Jordan type of $\alpha_{K}^{*}\left(M_{K} \otimes_{K} N_{K}\right)$ were maximal, then the second statement of Theorem 4.2 would imply that this maximal type is the same as that of $\alpha_{K}^{*}\left(M_{K}\right) \otimes_{K} \alpha_{K}^{*}\left(N_{K}\right)$. However, the hypotheses on $\alpha_{K}^{*}\left(M_{K}\right), \alpha_{K}^{*}\left(N_{K}\right)$ together with Proposition 4.1 would then lead to an immediate contradiction. 
We give two examples to illustrate that naturally formulated improvements of Theorem 4.2 are not valid. Our first example illustrates that the maximality of both $M$ and $N$ at a given $\pi$-point is not sufficient to imply the maximality of $M \otimes N$ at that $\pi$-point. Namely, we construct a module $W$ and a $\pi$-point $\beta_{L}$ such that $\beta_{L}$ has maximal Jordan type on $W$ but not on $W \otimes W$ (even though we have $\beta_{L}^{*}\left(W_{L} \otimes W_{L}\right) \simeq \beta_{L}^{*}\left(W_{L}\right) \otimes_{L} \beta_{L}^{*}\left(W_{L}\right)$ by Theorem 4.2). As usual, this anomaly comes from the fact that the ordering on Jordan types is not total.

Example 4.5. Let $G$ be a finite $p$-group which has two conjugacy classes of maximal elementary abelian subgroups, represented by $E$ and $E^{\prime}$ respectively. Furthermore, we require $E$ to be normal. Let $e=|E|, f=\frac{|G|}{|E|}$. Assume that $p>3$.

For example, take $G$ to be the $p$-Sylow subgroup of the wreath product $\mathbb{Z} / p \mathbb{Z} \imath S_{p}$, so that $G$ is isomorphic to $(\mathbb{Z} / p \mathbb{Z})^{p} \rtimes \mathbb{Z} / p \mathbb{Z}$. Then $G$ has two non-conjugate maximal elementary abelian $p$-subgroups: $E=(\mathbb{Z} / p \mathbb{Z})^{p}$ which is normal and

$$
F=(\mathbb{Z} / p \mathbb{Z} \times \mathbb{Z} / p \mathbb{Z} \times \cdots \times \mathbb{Z} / p \mathbb{Z})^{\mathbb{Z} / p \mathbb{Z}} \times \mathbb{Z} / p \mathbb{Z} \cong(\mathbb{Z} / p \mathbb{Z})^{\times 2} .
$$

By Quillen stratification, $\Pi(G)=X \cup Y$ where $X=\Pi(E) / G, Y=\Pi(F) / N_{G}(F)$. Let $\left[\alpha_{K}\right] \in X,\left[\beta_{L}\right] \in Y$ be generic points.

Choose a homogeneous cohomology class $\xi \in \mathrm{H}^{\bullet}(G, k)$ with the property that $\xi$ vanishes on $\left[\beta_{L}\right]$ but does not vanish on $\left[\alpha_{K}\right]$, and let $L_{\xi}$ be Carlson module which has the property that the support of $L_{\xi}$ is the zero locus of $\xi$. Set $M=\operatorname{Ind}_{E}^{G}\left(\Omega_{E}^{1}(k)\right)$, set $N=L_{\xi}^{\oplus n}$ for some positive integer $n$ which is to be determined, and set $W=M \oplus N$. It was shown in Example [21, 4.13], that if we pick $n$ to satisfy the inequality

$$
\frac{f}{p}<n<(p-1) \frac{f}{p}
$$

then the Jordan types $\alpha_{K}^{*}\left(W_{K}\right)$ and $\beta_{L}^{*}\left(W_{L}\right)$ are maximal, incomparable generic Jordan types of $W$.

Let $d=\operatorname{dim} W$. We proceed to deduce a condition on $n$ which would ensure the inequality

$$
\operatorname{JType}\left(\alpha_{K}^{*}\left(W_{K}^{\otimes 2}\right)\right)>\operatorname{JType}\left(\beta_{L}^{*}\left(W_{L}^{\otimes 2}\right)\right)
$$

By the Appendix, $[p-1] \otimes[p-1]=(p-2)[p]+1[1]$. Since $\alpha_{K}^{*}\left(W_{K}\right)=m[p]+f[p-1]$ for some $m$ (see [21, 4.13]), and has dimension $d$, we get

$$
\alpha_{K}^{*}\left(W_{K}^{\otimes 2}\right) \simeq\left(\alpha_{K}^{*}\left(W_{K}\right)\right)^{\otimes 2} \simeq\left(\frac{d^{2}-f^{2}}{p}\right)[p]+f^{2}[1] .
$$

Similarly, applying the decomposition of $\beta_{L}^{*}\left(W_{L}\right)$ obtained in [21, 4.13], we get

$$
\beta_{L}^{*}\left(W_{L}^{\otimes 2}\right) \simeq\left(\beta_{L}^{*}\left(W_{L}\right)\right)^{\otimes 2} \simeq\left(\frac{d^{2}-2 n^{2} p}{p}\right)[p]+2 n^{2}[p-1]+2 n^{2}[1] .
$$


In order for the Jordan type of $\alpha_{K}^{*}\left(W_{K}^{\otimes 2}\right)$ to dominate that of $\beta_{L}^{*}\left(W_{L}^{\otimes 2}\right)$ it suffices to choose $n$ such that $\alpha_{K}^{*}\left(W_{K}^{\otimes 2}\right)$ has more blocks of size $p$ than $\beta_{L}^{*}\left(W_{L}^{\otimes 2}\right)$ and fewer blocks altogether. In other words, we need for the following inequalities to hold:

$$
\frac{d^{2}-f^{2}}{p}>\frac{d^{2}-2 n^{2} p}{p}
$$

comparing the number of blocks of size $p$, and

$$
\frac{d^{2}-f^{2}}{p}+f^{2}<\frac{d^{2}-2 n^{2} p}{p}+4 n^{2}
$$

comparing the overall number of blocks. Simplifying, we get

$$
\begin{gathered}
f^{2}<2 n^{2} p, \\
(p-1) f^{2}<2 n^{2} p .
\end{gathered}
$$

Observe that the second inequality implies the first and simplifies to

$$
\sqrt{\frac{p-1}{2 p}} f<n
$$

Since $p$ is greater than 3 and divides $f$, it is possible to choose $n$ to satisfy

$$
\sqrt{\frac{p-1}{2 p}} f<n<\frac{p-1}{p} f .
$$

Since such $n$ automatically satisfies the inequality (9), we conclude that $W$ has maximal non-comparable types at $\left[\alpha_{K}\right],\left[\beta_{L}\right]$ but that the Jordan type of $\alpha_{K}^{*}\left(W_{K} \otimes_{K}\right.$ $\left.W_{K}\right)$ is strictly greater than that of $\beta_{L}^{*}\left(W_{L} \otimes_{L} W_{L}\right)$. Thus, $\beta_{L}$ has maximal Jordan type on $W$ but not on $W \otimes W$.

Our second example shows that the maximal Jordan type of $M \otimes N$ can occur at a $\pi$-point at which one of $M, N$ does not have maximal Jordan type and neither has projective type. This phenomenon can only occur if $\Pi(G)$ is reducible.

Example 4.6. As in the previous example, let $G$ be a finite group with exactly two conjugacy classes of maximal elementary abelian $p$-groups (e.g., the $p$-Sylow subgroup of the wreath product $\mathbb{Z} / p \mathbb{Z}$ ? $\left.S_{p}\right)$. Write $\Pi(G)=X \cup Y$ with $X, Y$ irreducible closed subsets, and let $\left[\alpha_{K}\right] \in X,\left[\beta_{L}\right] \in Y$ be generic points. Choose cohomology classes $\zeta, \xi \in \mathrm{H}^{\bullet}(G, k)$ such that

$$
\alpha_{K}^{*}\left(\zeta_{K}\right)=0 \neq \beta_{L}^{*}\left(\zeta_{L}\right), \quad \alpha_{K}^{*}\left(\xi_{K}\right) \neq 0=\beta_{L}^{*}\left(\xi_{K}\right) .
$$

Let $L_{\zeta}$ (respectively, $L_{\xi}$ ) be the corresponding Carlson module so that the support of $L_{\zeta}$ (resp., $L_{\xi}$ ) is the zero locus of $\zeta$ (resp., $\xi$ ). Recall that the Jordan type of $L_{\zeta}$ on its support is [proj] $+1[p-1]+1[1]$ for some $m$, and similarly for $L_{\xi}[21$, 4.8]. Let $M=L_{\zeta} \oplus L_{\xi}^{\oplus 2}$, so that $M$ has maximal Jordan type [proj] $+1[p-1]+1[1]$ at $\alpha_{K}$, and Jordan type [proj] $+2[p-1]+2[1]$ at $\beta_{L}$. Similarly, let $N=L_{\zeta}^{\oplus 2} \oplus L_{\xi}$, so that $N$ has Jordan type [proj] $+2[p-1]+2[1]$ at $\alpha_{K}$ and maximal Jordan type 
$[$ proj $]+1[p-1]+1[1]$ at $\beta_{L}$. Then $M \otimes N$ has the same Jordan type at both generic points $\alpha_{K}$ and $\beta_{L}$, so that this common generic Jordan type is maximal.

Corollary 4.4 enables us to prove the following property of the non-maximal support variety.

Proposition 4.7. Assume $\Pi(G)$ is irreducible and let $M, N$ be $k G$-modules. Then

$$
\Gamma(G)_{M \otimes N}=\left(\Gamma(G)_{M} \cup \Gamma(G)_{N}\right) \cap\left(\Pi(G)_{M} \cap \Pi(G)_{N}\right) .
$$

Proof. If $\alpha_{K}: K[t] / t^{p} \longrightarrow K G$ is a $\pi$-point such that either $\alpha_{K}^{*}\left(M_{K}\right)$ or $\alpha_{K}^{*}\left(N_{K}\right)$ is projective, then $\alpha_{K}^{*}\left(M_{K}\right) \otimes_{K} \alpha_{K}^{*}\left(N_{K}\right)$ is projective and thus of maximum type. Consequently, statement (1) of Theorem 4.2 implies that $\alpha_{K}^{*}\left(M_{K} \otimes_{K} N_{K}\right)$ is also projective and thus also maximum. For such $\alpha_{K},\left[\alpha_{K}\right] \notin \Gamma(G)_{M \otimes N}$. In other words, $\Gamma(G)_{M \otimes N} \subset \Pi(G)_{M} \cup \Pi(G)_{N}$.

Now suppose that $\alpha_{K}$ has maximal Jordan type on both $M$ and $N$. The statement (3) of Theorem 4.2 implies that $\alpha_{K}$ has maximal Jordan type on $M \otimes N$. Hence, $\Gamma(G)_{M \otimes N} \subset \Gamma(G)_{M} \cup \Gamma(G)_{N}$. We have established the inclusion

$$
\Gamma(G)_{M \otimes N} \subset\left(\Gamma(G)_{M} \cup \Gamma(G)_{N}\right) \cap\left(\Pi(G)_{M} \cap \Pi(G)_{N}\right) .
$$

On the other hand, assume that neither $\alpha_{K}^{*}\left(M_{K}\right)$ nor $\alpha_{K}^{*}\left(N_{K}\right)$ is projective, and that the Jordan type of either $\alpha_{K}^{*}\left(M_{K}\right)$ or $\alpha_{K}^{*}\left(N_{K}\right)$ is not maximal. In other words, we assume that $\left[\alpha_{K}\right] \in\left(\Gamma(G)_{M} \cup \Gamma(G)_{N}\right) \cap\left(\Pi(G)_{M} \cap \Pi(G)_{N}\right)$. Corollary 4.4 implies that $\alpha_{K}$ does not have maximal Jordan type on $M \otimes N$. Hence, $\left[\alpha_{K}\right] \in \Gamma(G)_{M \otimes N}$.

Remark 4.8. The previous examples show the necessity of the hypothesis of irreducibility in Proposition 4.7, Example 4.6 contradicts the inclusion

$$
\Gamma(G)_{M \otimes N} \supset\left(\Gamma(G)_{M} \cup \Gamma(G)_{N}\right) \cap\left(\Pi(G)_{M} \cap \Pi(G)_{N}\right),
$$

whereas Example 4.5 contradicts the inclusion

$$
\Gamma(G)_{M \otimes N} \subset\left(\Gamma(G)_{M} \cup \Gamma(G)_{N}\right) \cap\left(\Pi(G)_{M} \cap \Pi(G)_{N}\right) .
$$

Next we offer a suggestive characterization of modules of constant Jordan type for those finite group schemes $G$ with $\Pi(G)$ irreducible.

Proposition 4.9. If $\Pi(G)$ is irreducible, then a non-projective $k G$-module $M$ has constant Jordan type if and only if for every finite dimensional $k G$-module $N$ the tensor product $M \otimes N$ has the property that $\Gamma(G)_{N}=\Gamma(G)_{M \otimes N}$.

Proof. First assume that $M$ does not have constant Jordan type. If $N=k$ (the trivial module), then $\Gamma(G)_{k}$ is empty whereas $\Gamma(G)_{k \otimes M}$ is not empty.

Conversely, assume that $M$ has constant Jordan type and $M$ is not projective. Let $\alpha_{K}: K[t] / t^{p} \longrightarrow K G$ be a $\pi$-point such that $N$ has maximal Jordan type at $\alpha_{K}$. By Theorem 4.2, the Jordan type of $\alpha_{K}^{*}\left(M_{K} \otimes N_{K}\right) \simeq \alpha_{K}^{*}\left(M_{K}\right) \otimes \alpha_{K}^{*}\left(N_{K}\right)$ is maximal 
for $M \otimes N$. Hence, we get the inclusion $\Gamma(G)_{N} \supset \Gamma(G)_{M \otimes N}$. To prove the opposite inclusion, assume $\left[\alpha_{K}\right] \in \Gamma(G)_{N}$. Since $M$ is not projective, Corollary 4.4 implies that $\alpha_{K}$ does not have maximal Jordan type on $M \otimes N$. Hence, $\left[\alpha_{K}\right] \in \Gamma(G)_{M \otimes N}$, and we have established the opposite inclusion.

\section{Endotrivial modules}

We recall the definition of an endotrivial module, classically formulated for finite groups but admitting a natural extension to all finite group schemes. Endotrivial modules were introduced by Dade [12], who showed that for an abelian $p$-group, the only endotrivial $k G$-modules have the form $\Omega^{n}(k) \oplus P$ where $P$ is a projective module. The endotrivial modules are the building blocks for the endopermutation modules which for many groups are the sources of the simple modules and are also a part of the Picard group of self equivalences of the stable module category. See [9] for references. A classification of the endotrivial modules for finite $p$-groups was completed in [10].

Definition 5.1. Let $G$ be a finite group scheme over $k$. A $k G$-module $M$ is an endotrivial module provided $\operatorname{End}_{k}(M)$ is stably isomorphic as a $k G$-module to the trivial module. In other words, $M$ is endotrivial provided that there exists a $k G$ projective module $P$ and a $k G$-isomorphism

$$
\operatorname{Hom}_{k}(M, M) \cong k \oplus P .
$$

As can readily be verified (for example, using formula (10.2) of the Appendix) an indecomposable $k[t] / t^{p}$-module is endotrivial if and only if it is stably isomorphic to either $1[1]$ or $1[p-1]$, the trivial module $k$ or the Heller shift $\Omega^{1}(k)$ of $k$. More generally, Theorem 5.6 below implies that for any finite group scheme the Heller shifts $\Omega^{i}(k)$ of the trivial module are endotrivial modules. As mentioned earlier, for elementary abelian $p$-groups, these are the only indecomposable endotrivial modules. On the other hand, there do exist sporadic examples of finite groups admitting other endotrivial modules. For example, if $G$ is a dihedral group of order 8, then $\operatorname{rad}(k G) / \operatorname{rad}^{4}(k G)$ is the direct sum of two modules of dimension three that are endotrivial (see [9]).

As we show in Theorem 5.6 below, every endotrivial module is a module of constant Jordan type. As seen in section 2 and as well in the next section, there exist

many examples of modules of constant Jordan type which are not direct sums of endotrivial modules.

If $M, N$ are $k G$-modules, then we may identify $\operatorname{Hom}_{k}(M, N)$ as a $k G$-module with $M^{\#} \otimes N$, where $M^{\#}=\operatorname{Hom}_{k}(M, k)$. For our purposes, it suffices to analyze $M^{\#}$ and then apply Section 4 in order to investigate $\operatorname{Hom}_{k}(M, N)$.

Let $G$ be a finite group scheme and (as usual) let $k G$ denote the group algebra of $G$. Denote by $S$ the antipode of the Hopf algebra $k G$. If $\rho_{M}: k G \longrightarrow \operatorname{End}_{k}(M) \simeq$ 
$M^{\#} \otimes M$ is a finite dimensional representation of $G$ determining the $k G$-module $M$, then

$$
\rho_{M \#}=\phi \circ \rho_{M} \circ S: k G \longrightarrow k G \longrightarrow M^{\#} \otimes M \longrightarrow M \otimes M^{\#},
$$

where $\phi$ exchanges factors (and thus is the transpose from the point of view of matrices).

Observe that the dual $[i]^{\#}$ of the indecomposable $k[t] / t^{p}$-module $[i]$ is indecomposable, and thus isomorphic to $[i]$ as can be seen by comparing dimensions. The following proposition enables us to work with the generic and maximal Jordan types of $\operatorname{Hom}_{k}(M, N)$ for finite dimensional $k G$-modules $M, N$.

Proposition 5.2. Let $G$ be a finite group scheme, $M$ be a finite dimensional $k G$ module, and $\alpha_{K}: K[t] / t^{p} \longrightarrow K G$ be a $\pi$-point of $G$. Assume that $\alpha_{K}$ has maximal Jordan type on $M$. Then

$$
\alpha_{K}^{*}\left(M_{K}^{\#}\right) \simeq \alpha_{K}^{*}\left(M_{K}\right) \simeq\left(\alpha_{K}^{*}\left(M_{K}\right)\right)^{\#} .
$$

Moreover, $\alpha_{K}$ has maximal Jordan type on $M$ if and only if it has maximal Jordan type on $M^{\#}$.

Proof. Let $C_{K} \subset G_{K}$ be a unipotent abelian group scheme through which $\alpha_{K}$ factors, so that $\alpha_{K}$ has maximal Jordan type for $M_{K}$ as a $K C_{K}$-module. The restriction of the $K G$-module $M_{K}^{\#}$ to $K C_{K}$ is the dual of the restriction of $M_{K}$, since $K C_{K} \rightarrow K G_{K}$ is a map of Hopf algebras. Thus, to prove the first asserted isomorphism, it suffices to assume $G$ is a unipotent abelian finite group scheme.

Extending scalars if necessary so that $k$ is perfect, we get

$k G \simeq k\left[T_{1}, \ldots, T_{r}\right] /\left(T_{i}^{p^{e_{i}}}\right)($ see $[28,14.4])$. Let $t_{i}=T_{i}^{p^{e_{i}-1}}$. Let $I=\left(T_{1}, \ldots, T_{r}\right)$ be the augmentation ideal of $k G$, and let $I^{(p)}$ be the ideal generated by $\left(t_{1}, \ldots, t_{r}\right)$. Let $\nabla: k G \rightarrow k G \otimes k G$ be the coproduct in $k G$. Recall that $\nabla\left(\alpha_{K}(t)\right)-1 \otimes \alpha_{K}(t)-$ $\alpha_{K}(t) \otimes 1 \in I \otimes I\left(\left[23\right.\right.$, I.2.4]). Since $\alpha_{K}(t)$ has $p$-th power 0 , we can refine this further, concluding that

$$
\nabla\left(\alpha_{K}(t)\right)-1 \otimes \alpha_{K}(t)-\alpha_{K}(t) \otimes 1 \in I \otimes I^{(p)}+I^{(p)} \otimes I .
$$

Let $\mu: k G \otimes k G \rightarrow k G$ be the multiplication map in $k G$. We have $(\mu \circ(S \otimes$ Id) $)\left(\nabla\left(\alpha_{\mathrm{K}}(\mathrm{t})\right)=\epsilon\left(\alpha_{\mathrm{K}}(\mathrm{t})\right)=0\right.$ by one of the Hopf algebra axioms (see [23, I.2.3]) where $\epsilon: k G \rightarrow k$ is the counit map, and $S$ is the antipode. Hence, applying $\mu \circ(S \otimes \mathrm{Id})$ to $(111)$, we get

$$
\alpha_{K}(t)+S\left(\alpha_{K}(t)\right) \subset I \cdot I^{(p)}+I^{(p)} \cdot I .
$$

Moreover, we obtain the same inclusion if we change the base field from $K$ to any field extension $L$ over $K$. Therefore, we can apply [21, 1.12] to conclude that $\rho_{M}\left(\alpha_{K}(t)\right)$ and $\rho_{M}\left(S\left(\alpha_{K}(t)\right)\right)$ have the same (maximal) Jordan type. Hence, $\rho_{M}\left(\alpha_{K}(t)\right)$ and $\rho_{M}^{\#}\left(\alpha_{K}(t)\right)=\phi\left(\rho_{M}\left(S\left(\alpha_{K}(t)\right)\right)\right)$ have the same (maximal) Jordan type. We conclude that $\alpha_{K}^{*}\left(M_{K}^{\#}\right) \simeq \alpha_{K}^{*}\left(M_{K}\right)$. 
The second isomorphism follows immediately from the observation that $[i]^{\#}=[i]$.

Since sending $M$ to $M^{\#}$ is idempotent, we get the following corollary.

Corollary 5.3. Let $G$ be a finite group scheme, and let $M$ be a finite dimensional $k G$-module. Then

$$
\Gamma(G)_{M}=\Gamma(G)_{M^{\#}} .
$$

The next corollary follows immediately from Corollary 4.3, Proposition 5.2 and the isomorphism $\operatorname{Hom}_{k}(M, N) \simeq M^{\#} \otimes N$.

Corollary 5.4. Let $G$ be a finite group scheme, and let $M, N$ be finite dimensional $k G$-modules of constant Jordan types $\underline{a}=a_{p}[p]+\cdots+a_{1}[1], \underline{b}=b_{p}[p]+\cdots+b_{1}[1]$, respectively. Then $\operatorname{Hom}_{k}(M, N)$ has constant Jordan type $\underline{a} \otimes \underline{b}$ (given explicitly by the formula in the Appendix).

Corollary 5.5. Let $G$ be a finite group scheme, and consider two finite dimensional $k G$-modules $M, N$, and a $\pi$-point $\alpha_{K}$ of $G$. If $\alpha_{K}$ has maximal Jordan type on $\operatorname{Hom}(M, N)$, then

$$
\alpha_{K}^{*}\left(\operatorname{Hom}_{K}\left(M_{K}, N_{K}\right)\right) \simeq \operatorname{Hom}_{K}\left(\alpha_{K}^{*}\left(M_{K}\right), \alpha_{K}^{*}\left(N_{K}\right)\right)
$$

Proof. Let $i: C_{K} \hookrightarrow G_{K}$ be a unipotent abelian group scheme through which $\alpha_{K}$ factors, so that $\alpha_{K}$ is maximal on $\operatorname{Hom}_{K}\left(M_{K}, N_{K}\right)$ as a $K C_{K}$-module. Since $i: C_{K} \hookrightarrow G_{K}$ is a map of Hopf algebras, it commutes with Hom. Hence, we may assume that $G$ is a unipotent abelian group scheme. In particular, $\Pi(G)$ is irreducible.

Since $\alpha_{K}$ has maximal Jordan type on $\operatorname{Hom}(M, N) \simeq M^{\#} \otimes N$, Theorem 4.2(2) implies that

$$
\alpha_{K}^{*}\left(M_{K}^{\#} \otimes N_{K}\right) \simeq \alpha_{K}^{*}\left(M_{K}^{\#}\right) \otimes \alpha_{K}^{*}\left(N_{K}\right) .
$$

If $\alpha_{K}^{*}\left(M_{K}^{\#}\right) \otimes \alpha_{K}^{*}\left(N_{K}\right)$ is projective, then either $\alpha_{K}^{*}\left(N_{K}\right)$ or $\alpha_{K}^{*}\left(M_{K}^{\#}\right)$ is projective. Since projectivity of $\alpha_{K}^{*}\left(M_{K}^{\#}\right)$ implies projectivity of $\alpha_{K}^{*}\left(M_{K}^{\#}\right)$, we conclude that in this case $\operatorname{Hom}_{K}\left(\alpha_{K}^{*}\left(M_{K}\right), \alpha_{K}^{*}\left(N_{K}\right)\right)$ is projective.

Assume that $\alpha_{K}^{*}\left(M_{K}^{\#}\right) \otimes \alpha_{K}^{*}\left(N_{K}\right)$ is not projective. In this case neither $\alpha_{K}^{*}\left(N_{K}\right)$ nor $\alpha_{K}^{*}\left(M_{K}^{\#}\right)$ is projective. Since $\Pi(G)$ is irreducible, Corollary 4.4 implies that $\alpha_{K}$ has maximal Jordan types on both $N$ and $M^{\#}$. Hence, $\alpha_{K}^{*}\left(M_{K}^{\#}\right) \simeq\left(\alpha_{K}^{*}\left(M_{K}\right)\right)^{\#}$. Therefore, $\operatorname{Hom}_{K}\left(\alpha_{K}^{*}\left(M_{K}\right), \alpha_{K}^{*}\left(N_{K}\right)\right) \simeq\left(\alpha_{K}^{*}\left(M_{K}\right)\right)^{\#} \otimes \alpha_{K}^{*}\left(N_{K}\right) \simeq \alpha_{K}^{*}\left(M_{K}^{\#}\right) \otimes$ $\alpha_{K}^{*}\left(N_{K}\right) \simeq \alpha_{K}^{*}\left(\operatorname{Hom}_{K}\left(M_{K}, N_{K}\right)\right)$.

We now conclude that endotrivial modules are modules of constant Jordan type. The second statement of the theorem provides a "local" criterion of endotriviality, similar to the projectivity criterion given by the Dade's lemma (see [12]). 
Theorem 5.6. Let $G$ be a finite group scheme, and let $M$ be a finite dimensional $k G$-module.

(1) If $M$ is endotrivial, then $M$ has constant Jordan type of the form either $m[p]+1[1]$ or $m[p]+1[p-1]$ for some $m \geq 0$, and thus $\alpha_{K}^{*}\left(M_{K}\right)$ is endotrivial for every $\pi$-point $\alpha_{K}$ of $G$.

(2) Conversely, if $\alpha_{K}^{*}\left(M_{K}\right)$ is endotrivial for each $\pi$-point $\alpha_{K}$ of $G$ (and hence of the form either $m[p]+1[1]$ or $m[p]+1[p-1])$, then $M$ is endotrivial.

Proof. Observe that any endotrivial module must have dimension whose square is congruent to 1 modulo $p$ and thus must have dimension congruent to either 1 or $p-1$ modulo $p$. To prove the first assertion, we assume that $M$ is endotrivial, so that $\operatorname{End}_{k}(M)=k \oplus(\operatorname{proj})$. Thus, $\alpha_{K}^{*}\left(\operatorname{End}_{K}\left(M_{K}\right)\right)$ has Jordan type $m[p]+1[1]$ at each $\pi$-point $\alpha_{K}$. In particular, every $\pi$-point $\alpha_{K}$ has maximal Jordan type on $\operatorname{End}_{k}(M)$. By Corollary [5.5, $\alpha_{K}^{*}\left(\operatorname{End}_{K}\left(M_{K}\right)\right) \simeq \operatorname{End}_{K}\left(\alpha_{K}^{*}\left(M_{K}\right)\right)$. Hence, $\alpha_{K}^{*}\left(M_{K}\right)$ is an endotrivial $K[t] / t^{p}$-module. The statement now follows from the fact that the only such modules are of the form $m[p]+1[1]$ or $m[p]+1[p-1]$ for some $m \geq 0$.

To prove the converse, we assume that $\alpha_{K}^{*}\left(M_{K}\right)$ is an endotrivial $K[t] / t^{p}$-module for each $\pi$-point $\alpha_{K}: K[t] / t^{p} \longrightarrow K G$. Thus, for each $\alpha_{K}, \alpha_{K}^{*}\left(M_{K}\right)$ has Jordan type either $m[p]+1[1]$ or $m[p]+1[p-1]$ for some $m \geq 0$. Since the dimension of $M$ can not be congruent to both 1 and $p-1$ modulo $p$, we conclude that $M$ has constant Jordan type. Consider the short exact sequence

$$
0 \longrightarrow X \longrightarrow \operatorname{End}_{k}(M) \stackrel{\operatorname{Tr}}{\longrightarrow} k \longrightarrow 0,
$$

where $\operatorname{Tr}$ is the trace map. By Corollary 5.5, $\alpha_{K}^{*}\left(\operatorname{End}_{K}\left(M_{K}\right)\right) \simeq \operatorname{End}_{K}\left(\alpha_{K}^{*}\left(M_{K}\right)\right)$. Moreover, because the dimension of $\operatorname{End}_{k}(M)$ is relatively prime to $p$, the trace map of (12) splits. Pulling back the split short exact sequence (12) via $\alpha_{K}$, we conclude that

$$
\alpha_{K}^{*}(X) \simeq \operatorname{ker}\left\{\operatorname{End}_{K}\left(\alpha_{K}^{*}\left(M_{K}\right)\right) \longrightarrow K\right\} \simeq m[p]
$$

is projective for all $\pi$-points $\alpha_{K}$ and thus $X$ is projective $([20,5.4])$. Hence, $M$ is endotrivial.

\section{Constructing modules of Constant Jordan type}

In this section, we consider two different methods of constructing modules of constant Jordan type. Proposition 6.1 presents the observation that an extension of modules of constant Jordan type has "total module" also of constant Jordan type if the extension splits when pulled back along any $\pi$-point. This observation fits well with the Auslander-Reiten theory of almost split exact sequences as we discuss in §8. Proposition 6.6 presents a method of constructing extensions of constant Jordan type whose pull-backs along $\pi$-points are not split. 
We shall frequently utilize the Tate cohomology ring $\widehat{\mathrm{H}}^{*}(G, k)$ for a finite group scheme $G$, and The Tate Ext groups $\widehat{\operatorname{Ext}}^{*}(M, N)$ for $G$-modules $M, N$. Tate cohomology ring $\widehat{\mathrm{H}}^{*}(G, k)$ is a graded commutative $k$-algebra which coincides with regular cohomology in positive degrees. One advantage of Tate cohomology that we will exploit throughout this section is that it allows for arbitrary degree shifts. Namely, we have the following formulas

$$
\begin{gathered}
\widehat{\operatorname{Ext}}^{n}(M, N) \simeq \widehat{\operatorname{Ext}}^{0}\left(\Omega^{n} M, N\right)=\operatorname{Hom}_{\operatorname{stmod}(k G)}\left(\Omega^{n} M, N\right) \simeq \\
\operatorname{Hom}_{\operatorname{stmod}(k G)}\left(M, \Omega^{-n} N\right)=\widehat{\operatorname{Ext}}^{0}\left(M, \Omega^{-n} N\right)
\end{gathered}
$$

for any $n \in \mathbb{Z}$. We refer the reader to [3] for further details.

Proposition 6.1. Suppose that $G$ is a finite group scheme over $k$. Let $M$ and $N$ be $k G$-modules of constant Jordan type, and suppose that

$$
0 \longrightarrow M \longrightarrow B \longrightarrow N \longrightarrow 0
$$

is an exact sequence. Let $\zeta \in \operatorname{Ext}_{k G}^{1}(N, M)$ be the class of (13). If for every $\pi$-point $\alpha_{K}: K[t] / t^{p} \longrightarrow K G$ the restriction $\alpha_{K}^{*}(\zeta)$ is zero, then $B$ has constant Jordan type. Moreover, if the Jordan types of $M$ and $N$ are $\sum_{i=1}^{p} m_{i}[i]$ and $\sum_{i=1}^{p} n_{i}[i]$, then the Jordan type of $B$ is $\sum_{i=1}^{p}\left(m_{i}+n_{i}\right)[i]$.

Proof. Because the cohomology class vanishes under restriction to a $\pi$-point $\alpha_{K}$, we have that the restriction of (13) along $\alpha_{K}$ splits and $\alpha_{K}^{*}\left(B_{K}\right) \simeq \alpha_{K}^{*}\left(M_{K}\right) \oplus \alpha_{K}^{*}\left(N_{K}\right)$. The result is now obvious.

Proposition 6.1 does not always produce "new" examples of modules of constant Jordan type as we observe in the special case $G=\mathbb{Z} / 2 \mathbb{Z} \times \mathbb{Z} / 2 \mathbb{Z}$.

Example 6.2. Let $G$ be $\mathbb{Z} / 2 \mathbb{Z} \times \mathbb{Z} / 2 \mathbb{Z}$. As presented in [2], [22], there is a complete classification of the $k G$-modules. Using this classification, we observe that the only indecomposable $k G$-modules of constant Jordan type are of the form $\Omega^{n}(k)$ for some $n$.

Namely, it is shown in [22] that all of the indecomposable $k G$-modules of odd dimension are of the form $\Omega^{n}(k)$ for some $n$. On the other hand, the non-projective indecomposable modules of even dimension are all isomorphic to $L_{\zeta}$ for $\zeta \in \mathrm{H}^{n}(G, k)$. The support varieties of these even dimensional modules are proper non-trivial subvarieties of $\Pi(G)$, so that none of these have constant Jordan type.

It is instructive to look more closely to see why Proposition 6.1 does not determine other modules of constant Jordan type in this example. Observe that $\mathrm{H}^{*}(G, k) \cong \operatorname{Ext}_{k G}^{*}(k, k)$ is a polynomial ring in two variables having no non-zero element whose restriction to every $\pi$-point vanishes. Hence the only possible application of Proposition 6.1 would be in a situation where $N \cong \Omega^{n}(k), M \cong \Omega^{m}(k)$ and $n<m$. Then (13) represents an element of negative Tate cohomology, $\zeta$ of $\widehat{\mathrm{H}}^{n-m+1}(G, k)$. 
By Proposition 6.3 which follows, $\zeta$ restricts to zero at every $\pi$-point, but the middle term of this non-split short exact sequence splits as $\Omega^{n+a}(k) \oplus \Omega^{n+b} \oplus$ (proj) where $a$ and $b$ are nonnegative integers such that $a+b=m-n$ (see the proof of Theorem 6.13 .

The preceding example is special since $\mathbb{Z} / 2 \mathbb{Z} \times \mathbb{Z} / 2 \mathbb{Z}$ has tame representation type. For more general groups, the observation of Proposition 6.1 in conjunction with the following proposition does give new examples.

Proposition 6.3. Let $G$ be a finite group scheme with the property that every $\pi$ point factors by way of a flat map through a unipotent abelian group scheme whose cohomology has Krull dimension at least 2. Let $\zeta \in \widehat{\mathrm{H}}^{n}(G, k)$ for $n<0$ be an element in negative Tate cohomology of $G$ corresponding to a short exact sequence of the form

$$
0 \longrightarrow k \longrightarrow E \longrightarrow \Omega^{n-1}(k) \longrightarrow 0
$$

Then for any $\pi$-point $\alpha_{K}: K[t] / t^{p} \longrightarrow K G$, the pull-back of (14) along $\alpha_{K}$ is split (i.e., $\left.\alpha_{K}^{*}\left(\zeta_{K}\right)=0\right)$.

Proof. Recall that every element $\zeta$ of $\widehat{\mathrm{H}}^{n}(G, k)$ is represented by a homomorphism $\zeta^{\prime}: k \longrightarrow \Omega^{-n}(k)$. In order to prove the lemma, it is sufficient to show that for any $\pi$-point $\alpha_{K}: K[t] /\left(t^{p}\right) \longrightarrow K G$, the restriction of $\zeta^{\prime}$ factors through a projective $K[t] /\left(t^{p}\right)$-module. We proceed to establish such a factorization using our knowledge of the modules $\Omega^{-n}(K)$ for $n<0$. By Theorem 1.4, equivalent $\pi$-points induce the same map in cohomology. Thus, it suffices to prove the statement for some representative $\alpha_{K}$ of each $\left[\alpha_{K}\right] \in \Pi(G)$.

Our hypothesis immediately allows us to replace $G$ by some abelian unipotent group scheme (defined over $K$ ) whose cohomology has Krull dimension at least 2. After possibly passing to some finite extension of $K,[28,14.1]$ enables us to conclude that $K G \simeq K\left[T_{1}, \ldots, T_{r}\right] /\left(T_{1}^{p^{e_{1}}}, \ldots, T_{r}^{p^{e_{r}}}\right)$, where $r \geq 2$. Because neither our hypothesis nor our conclusion depends upon the coalgebra structure on $K G$, we may assume that $G$ is an abelian $p$-group. Let $t_{i}=T_{i}^{p^{e_{i}-1}}$ and let $E \subset G$ be the (unique) elementary abelian $p$-group with $K E=k\left[t_{1}, \ldots, t_{r}\right] /\left(t_{1}^{p}, \ldots, t_{r}^{p}\right) \subset K G$. By $[18,4.1]$, any $K$-rational $\pi$-point of $K G$ has a representative factoring through $K E$. Thus, we may assume that $G=E$ is an elementary abelian $p$-group of rank at least 2. Changing the generators of $K E$, we may further assume $t_{1}=\alpha_{K}(t)$; moreover, it suffices to assume that $E$ has rank 2 , for if the proposition is valid for an elementary abelian subgroup of $G$ of rank 2 , then it is valid for $G$ itself. Thus, we are reduced to the case that $K G$ is isomorphic to $K[u, v] /\left(u^{p}, v^{p}\right)$ with $u=\alpha_{K}(t)$.

The structure of a minimal $K G$-projective resolution $P_{\bullet} \rightarrow K$ is well known [11], with $P_{m-1}=K G^{\times m}$. A set of generators $a_{1}, \ldots, a_{m}$ for $P_{m-1}$ can be chosen so that 
$\Omega^{m}(K)$ is the submodule generated by the elements

$$
u^{p-1} a_{1}, \quad v a_{1}-u a_{2}, \quad v^{p-1} a_{2}-u^{p-1} a_{3}, \quad v a_{3}-u a_{4}, \quad \ldots, \quad v a_{m-1}-u a_{m}, \quad v^{p-1} a_{m}
$$

for $m$ even and

$$
u a_{1}, \quad v a_{1}-u^{p-1} a_{2}, \quad v^{p-1} a_{2}-u a_{3}, \quad v a_{3}-u^{p-1} a_{4}, \quad \ldots, \quad v^{p-1} a_{m-1}-u a_{m}, \quad v a_{m}
$$

for $m$ odd. Every $K G$-fixed point of $P_{m-1}$, and thus also of $\Omega^{m}(K)$ is a linear combination of the elements $u^{p-1} v^{p-1} a_{i}$. Moreover, for $i$ odd and $m$ even,

$$
u^{p-1} v^{p-1} a_{i}=u^{p-1} v^{p-2}\left(v a_{i}-u a_{i+1}\right)
$$

and for $i$ even and $m$ even,

$$
u^{p-1} v^{p-1} a_{i}=u^{p-1}\left(v^{p-1} a_{i}-u^{p-1} a_{i+1}\right) .
$$

Similar formulas hold for $m$ odd. Consequently, every $K G$-fixed point of $\Omega^{m}(K)$ has the form $u^{p-1} z$ for some $z \in \Omega^{m}(K)$. Thus, as a map of $K[t] / t^{p}$-modules (via $\left.\alpha_{K}: K[t] / t^{p} \longrightarrow K G\right), \zeta_{K}^{\prime}$ factors as the composition of $K \longrightarrow K[t] / t^{p}$ sending 1 to $t^{p-1}$ and $K[t] / t^{p} \longrightarrow \Omega^{m}(K)$ sending 1 to $z$. Hence, $\alpha_{K}^{*}\left(\zeta^{\prime}\right)$ factors through the rank 1 projective module $K[t] / t^{p}$ as required.

The following property is an immediate corollary of Proposition 6.3.

Corollary 6.4. Let $E$ be an elementary abelian p-group of rank at least 2, and let

$$
\theta: \Omega^{m}(k) \longrightarrow \Omega^{n}(k)
$$

be a homomorphism. If the restriction of $\theta$ along some $\pi$-point $\alpha_{K}: K[t] / t^{p} \rightarrow K E$ does not factor through a projective $K[t] / t^{p}$-module, then $m \geq n$.

The following proposition shows that there are limitations on the Jordan types which can be realized as extensions.

Proposition 6.5. Let $G$ be a finite group scheme over a field $k$ of characteristic $p>2$ with the property that every $\pi$-point factors by way of a flat map through a unipotent abelian group scheme whose cohomology has Krull dimension at least 2. Then there does not exist a $k G$-module $M$ of constant Jordan type $n[p]+1[2]$ which is an extension of a $k G$-module of constant Jordan type $m[p]+1[1]$ and one of constant Jordan type $(n-m)[p]+1[1]$.

Proof. As argued above in the proof of Proposition 6.3. we may assume that $G$ is an elementary abelian $p$-group of rank 2 . Consider a short exact sequence of the form

$$
E: \quad 0 \longrightarrow L \longrightarrow M \longrightarrow N \longrightarrow 0
$$

in which both $L$ and $N$ have stable constant Jordan type 1[1]. By Theorem 5.6, $L$ and $N$ are endotrivial modules, so that $L \cong \Omega^{a}(k), N \cong \Omega^{b}(k)$ for some integers $a$ and $b$. Since $p>2$, both $a$ and $b$ must be even integers. 
Thus the class of (15) is a cohomology class $\zeta$ in

$$
\operatorname{Ext}_{k G}^{1}\left(\Omega^{b}(k), \Omega^{a}(k)\right) \cong \widehat{\mathrm{H}}^{b-a+1}(G, k) .
$$

If $M$ has constant Jordan type $n[p]+1[2]$, then the restriction of (15) along any $\pi$-point $\alpha_{K}$ does not split; equivalently, the restricted class $\alpha_{K}^{*}(\zeta)$ does not vanish. However, the only such cohomology classes $\zeta$ are scalar multiples of the identity in degree $b-a+1=0$. Since both $a$ and $b$ are even integers, such classes $\zeta$ can not occur.

We now proceed to describe a second method of constructing modules of stable constant Jordan type $n[1]$ that cannot be created by the methods of Proposition 6.1. The construction can be summarized as follows: if a certain map represented by a matrix with coefficients in $\widehat{\mathrm{H}}^{*}(G, k)$ has the maximal possible rank when restricted to any $\pi$-point of $G$, then it has a kernel of constant Jordan type.

Theorem 6.6. Let $G$ be a finite group scheme, and choose integers $m>n, m_{i}$ and $n_{j}$ such that all $m_{i}$ and $n_{j}$ are even if $p>2$. Choose cohomology classes $\zeta_{i, j} \in \widehat{\mathrm{H}}^{m_{j}-n_{i}}(G, k)$ and let $\hat{\zeta}_{i, j}: \Omega^{m_{j}}(k) \rightarrow \Omega^{n_{i}}(k)$ represent $\zeta_{i, j}$. We consider an exact sequence

$$
0 \longrightarrow L \longrightarrow M \stackrel{\varphi=\left(\hat{\zeta}_{i, j}\right)}{\longrightarrow} N \longrightarrow 0
$$

of $k G$-modules where

$$
M \cong \sum_{j=1}^{m} \Omega^{m_{j}}(k) \oplus(\text { proj }) \quad \text { and } \quad N \cong \sum_{i=1}^{n} \Omega^{n_{i}}(k) .
$$

Assume that for every $\pi$-point $\alpha_{K}: K[t] / t^{p} \longrightarrow K G$, the restriction of the matrix of cohomology elements $\left(\alpha_{K}^{*}\left(\zeta_{i, j}\right)\right) \in M_{n, m}\left(\widehat{\mathrm{H}}^{*}\left(K[t] / t^{p}, K\right)\right)$ has rank $n$. Then the module $L$ has stable constant Jordan type $(m-n)[1]$.

Proof. Let $\alpha_{K}: K[t] / t^{p} \longrightarrow K G$ be a $\pi$-point. For any $s$, which is even if $p>2$, the restriction of the module $\Omega^{s}(k)$ along $\alpha_{K}$ has the form $\alpha_{K}^{*}\left(\Omega^{s}(K)\right) \cong K \oplus($ proj $)$. Moreover, if we have a map $\zeta: \Omega^{s}(k) \longrightarrow \Omega^{t}(k)$ whose cohomology class when restricted along $\alpha_{K}$ is not zero, then the composition

$$
K \stackrel{\iota}{\longrightarrow} \alpha_{K}^{*}\left(\Omega^{s}(K)\right) \stackrel{\alpha_{K}^{*}\left(\zeta_{K}\right)}{\longrightarrow} \alpha_{K}\left(\Omega^{t}(K)\right) \stackrel{\rho}{\longrightarrow} K,
$$

where $\iota$ and $\rho$ are the split inclusion and projection maps, is an isomorphism. Thus our hypothesis asserts that the composition

$$
K^{m} \stackrel{\iota}{\longrightarrow} \alpha_{K}^{*}\left(M_{K}\right) \stackrel{\alpha^{*}\left(\varphi_{K}\right)}{\longrightarrow} \alpha_{K}^{*}\left(N_{K}\right) \stackrel{\rho}{\longrightarrow} K^{n}
$$


has rank $n$. Since $\alpha_{K}^{*}\left(M_{K}\right)=(\operatorname{proj})+m[1]$ and $\alpha_{K}^{*}\left(N_{K}\right)=(\operatorname{proj})+n[1]$, it follows that the kernel of $\varphi, \alpha_{K}^{*}(L)$, has stable Jordan type $(m-n)[1]$. As this happens for any $\alpha_{K}$, we are done.

Theorem 6.6 is stated for even dimensional cohomology classes (for $p>2$ ), yet as we observe in the following proposition a very similar argument can also be applied to odd dimensional classes to yield additional modules of constant Jordan type.

Proposition 6.7. Let $G$ be a finite group scheme, and choose positive integers $m>n, m_{1}, \ldots m_{m}$ all odd $, n_{1}, \ldots, n_{n}$ all even. Assume $p>2$. Choose cohomology classes $\zeta_{i, j} \in \widehat{\mathrm{H}}^{m_{j}-n_{i}}(G, k)$ and let $\hat{\zeta}_{i, j}: \Omega^{m_{j}}(k) \rightarrow \Omega^{n_{i}}(k)$ represent $\zeta_{i, j}$. We consider an exact sequence

$$
0 \longrightarrow L \longrightarrow M \stackrel{\varphi=\left(\hat{\zeta}_{i, j}\right)}{\longrightarrow} N \longrightarrow 0
$$

of $k G$-modules where

$$
M \cong \sum_{j=1}^{m} \Omega^{m_{j}}(k) \oplus(\operatorname{proj}) \quad \text { and } \quad N \cong \sum_{i=1}^{n} \Omega^{n_{i}}(k) .
$$

Assume that for every $\pi$-point $\alpha_{K}: K[t] /\left(t^{p}\right) \longrightarrow K G$, the restriction of the matrix of cohomology elements $\alpha_{K}^{*}\left(\zeta_{i, j}\right) \in M_{n, m}\left(\widehat{\mathrm{H}}^{*}\left(K[t] / t^{p}, K\right)\right)$ has rank $n$. Then the module $L$ has stable constant Jordan type $(m-n)[p-1]+n[p-2]$.

Proof. Our hypothesis implies that the restriction of $\left(\hat{\zeta}_{i, j}\right)$ via any $\alpha_{K}$ is a map of $K[t] / t^{p}$-modules which remains surjective after free summands are dropped. We write such a map of $K[t] / t^{p}$-modules symbolically as a map of their (stable) Jordan types $m[p-1] \rightarrow n[1]$. Such a surjective map of $K[t] / t^{p}$-modules with indicated stable Jordan type necessarily has kernel with Jordan type $(m-n)[p-1]+n[p-2]$.

In general, it is very easy to construct examples for which Theorem 6.6 and Proposition 6.7 are relevant. These modules are multi-parametrized versions of important modules introduced and studied by the first author.

Example 6.8. Let $\xi_{1} \ldots, \xi_{r}$ be homogeneous elements in $\mathrm{H}^{\bullet}(G, k)$ such that the radical of the ideal generated by the $\xi_{i}$ 's is the augmentation ideal of $\mathrm{H}^{\bullet}(G, k)$. Alternatively, for $p$ odd, let $\xi_{1} \ldots, \xi_{r}$ be homogeneous elements in $\mathrm{H}^{*}(G, k)$ of odd degree whose Bocksteins generate an ideal of $\mathrm{H}^{\bullet}(G, k)$ whose radical is the augmentation ideal. For each $i$, let $\hat{\xi}_{i}: \Omega^{n_{i}}(k) \longrightarrow k$ be a cocycle representing $\xi_{i}$, where $n_{i}$ is the degree of $\xi_{i}$. Define

$$
\varphi: \bigoplus_{i=1}^{r} \Omega^{n_{i}}(k) \rightarrow k
$$


by the formula $\varphi\left(a_{1}, \ldots, a_{r}\right)=\hat{\xi}_{1}\left(a_{1}\right)+\cdots+\hat{\xi}_{r}\left(a_{r}\right)$. The condition that either the $\xi_{i}$ 's or the Bockstein's of the $\xi_{i}$ 's generate an ideal of $\mathrm{H}^{\bullet}(G, k)$ whose radical is the augmentation ideal implies that for each $\pi$-point $\alpha_{K}$, there is some $i$ such that $\alpha_{K}^{*}(\hat{\xi}) \neq 0$. (Namely, no point $\left[\alpha_{K}\right] \in \Pi(G) \cong \operatorname{Proj} \mathrm{H}^{\bullet}(G, k)$ lies in the union of the zero loci of either the $\xi_{i}$ 's or the Bockstein's of the $\xi_{i}$ 's.) Thus,

$$
L_{\xi_{1}, \ldots, \xi_{r}} \equiv \operatorname{Ker} \varphi
$$

has constant Jordan type.

In Theorem 6.13 below, we give an explicit example of such an $L_{\xi_{1}, \ldots, \xi_{r}}$ which can not be constructed using the technique of Proposition 6.1. The detailed verification of this example will occupy the remainder of this section, and involves the following four lemmas.

Recall that if $E \simeq(\mathbb{Z} / p \mathbb{Z})^{\times r}$ is an elementary abelian $p$-group of rank $r$, then

$$
\mathrm{H}^{*}(E, k) \cong \begin{cases}k\left[\zeta_{1}, \ldots, \zeta_{r}\right] \otimes \Lambda\left(\eta_{1}, \ldots, \eta_{r}\right) & \text { if } \quad p>2 \\ k\left[\eta_{1}, \ldots, \eta_{r}\right] & \text { if } \quad p=2 .\end{cases}
$$

where each $\eta_{j}$ has degree one and each $\zeta_{i}$ has degree 2 . Here, $\Lambda\left(\eta_{1}, \ldots, \eta_{r}\right)$ is the exterior algebra on the generators $\eta_{1}, \ldots, \eta_{r}$.

Lemma 6.9. Let $E$ be an elementary abelian p-group of rank $r$, for $r>1$. For $n>0$,

(1) $\operatorname{dim} \mathrm{H}^{n}(E, k)=\left(\begin{array}{c}n+r-1 \\ r-1\end{array}\right)$.

(2) $\operatorname{dim} P_{n}=\left(\begin{array}{c}n+r-1 \\ r-1\end{array}\right) \cdot p^{r}$, where $P_{n}$ is the $n$-th term of a minimal $k E$-projective resolution of $k$.

(3) $\operatorname{dim} \Omega^{n}(k)=p^{r} \cdot a_{r, n}+(-1)^{n}, n>0$ where

$$
a_{r, n}=\left(\begin{array}{c}
n+r-2 \\
r-1
\end{array}\right)-\left(\begin{array}{c}
n+r-3 \\
r-1
\end{array}\right)+\cdots+(-1)^{n-1}\left(\begin{array}{c}
r-1 \\
r-1
\end{array}\right) .
$$

(4) If $r=2$, then $\operatorname{dim}\left(\Omega^{2 n}(k)\right)=p^{2} n+1$.

(5) If $r=3$, then $\operatorname{dim}\left(\Omega^{2 n}(k)\right)=p^{3} n(n+1)+1$

Finally, if $n<0$, then $\operatorname{dim}\left(\Omega^{n}(k)\right)=\operatorname{dim}\left(\Omega^{-n}(k)\right)$.

Proof. The computation of $\operatorname{dim} \mathrm{H}^{n}(E, k)$ is a straightforward and familiar computation. In a minimal projective $k E$-resolution of $k$,

$$
\cdots \longrightarrow P_{2} \stackrel{\partial_{2}}{\longrightarrow} P_{1} \stackrel{\partial_{1}}{\longrightarrow} P_{0} \stackrel{\varepsilon}{\longrightarrow} k \longrightarrow 0
$$

each $P_{n}$ is a direct sum of copies of $k E$, the number of copies equal to the dimension of $\mathrm{H}^{n}(E, k)$. Using the vanishing of the Euler characteristic of an exact sequence, we conclude the asserted formula for the dimension of $\Omega^{n}(k)$. The assertions for $r=2,3$ are special cases. 
Lemma 6.10. Let $E$ be an elementary abelian p-group of rank $3, n$ be a negative integer, and $Q \longrightarrow \Omega^{2 n}(k)$ be the projective cover of the $2 n^{\text {th }}$ Heller shift of $k$. Then $\operatorname{dim} Q=p^{3}\left(2 n^{2}-n\right)$.

Proof. Taking duals we have an injection $\Omega^{-2 n}(k) \longrightarrow Q^{*}$, where $Q^{*}$ is the injective hull of $\Omega^{-2 n}(k)$. Hence, $Q^{*} \simeq P_{-2 n-1}$, the $(-2 n-1)^{\text {st }}$ term of the minimal projective resolution of $k$. As seen in Lemma 6.9 (2), the dimension of $P_{-2 n-1}$ is $p^{3}\left(\begin{array}{c}-2 n-1+2 \\ 2\end{array}\right)=p^{3}\left(2 n^{2}-n\right)$.

Lemma 6.11. Suppose that $E$ is an elementary abelian p-group of rank at least 2. Let

$$
\theta: \Omega^{m}(k) \longrightarrow \Omega^{n}(k)
$$

be a homomorphism for some nonnegative integers $m$ and $n$, which are both even in case $p>2$. Assume that for some $\pi$-point $\alpha_{K}: K[t] /\left(t^{p}\right) \longrightarrow K E$ the restriction along $\alpha$ of $\theta$ does not factor through a projective $K[t] /\left(t^{p}\right)$-module. Then $\theta$ is surjective.

Proof. By Corollary 6.4, we have $m-n \geq 0$. Let $\widehat{\theta}$ denote the cohomology class of $\theta$ in

$$
\operatorname{Ext}_{k E}^{m-n}(k, k) \cong \mathrm{H}^{m-n}(E, k) .
$$

The condition on the restriction of $\theta$ along $\alpha$ together with the fact that $m-n$ is even if $p>2$ implies that $\widehat{\theta}$ is a non-nilpotent element of $\mathrm{H}^{m-n}(E, k)$. Hence, multiplication by $\widehat{\theta}$ induces an injective map $\mathrm{H}^{n}(E, k) \longrightarrow \mathrm{H}^{m}(E, k)$. Since $\operatorname{Hom}_{k E}\left(\Omega^{\ell}(k), k\right) \cong$ $\mathrm{H}^{\ell}(E, k)$ for $\ell \geq 0$, it follows that $\theta$ induces an injective map

$$
\theta^{\prime}: \operatorname{Hom}_{k E}\left(\Omega^{n}(k), k\right) \longrightarrow \operatorname{Hom}_{k E}\left(\Omega^{m}(k), k\right) .
$$

So $\theta$ must be surjective.

Lemma 6.12. Suppose that $E$ is an elementary abelian p-group of rank 2. Let $\xi_{1} \in \mathrm{H}^{2 m}(E, k), \xi_{2} \in \mathrm{H}^{2 n}(E, k)$, and assume that the radical of the ideal generated by $\xi_{1}, \xi_{2}$ is the augmentation ideal of $\mathrm{H}^{\bullet}(E, k)$. Consider the exact sequence

$$
0 \longrightarrow L_{\xi_{1}, \xi_{2}} \longrightarrow \Omega^{2 m}(k) \oplus \Omega^{2 n}(k) \stackrel{\left(\begin{array}{l}
\xi_{1} \\
\xi_{2}
\end{array}\right)}{\longrightarrow} k \longrightarrow 0 .
$$

Then $L_{\xi_{1}, \xi_{2}} \simeq \Omega^{2 m+2 n}(k)$.

Proof. The condition on $\xi_{1}, \xi_{2}$ is equivalent to the condition that the matrix $\left(\xi_{1}, \xi_{2}\right)$ has rank 1 when restricted to any $\pi$-point of $G$. Hence, by Theorem $6.6, L_{\xi_{1}, \xi_{2}}$ has stable constant Jordan type 1[1], and it is an endotrivial module. By Lemma 6.9, the dimension of $L_{\xi_{1}, \xi_{2}}$ is $p^{2}(m+n)+1$, and hence $L_{\xi_{1}, \xi_{2}} \simeq \Omega^{ \pm(2 m+2 n)}(k)$. 
Now suppose that $L_{\xi_{1}, \xi_{2}} \simeq \Omega^{-(2 m+2 n)}(k)$. Then the sequence represents a nonzero element $\gamma \in \mathrm{H}^{2 m+2 n+1}(E, k)$ which has the property that $\alpha_{K}^{*}\left(\gamma_{K}\right)$ is zero for

any $\pi$-point $\alpha_{K}: K[t] / t^{p} \longrightarrow K E$. However, because $2 m+2 n+1$ is both positive and odd, there is no such element. Therefore, we must have that $L_{\xi_{1}, \xi_{2}} \simeq \Omega^{2 m+2 n}(k)$ as desired.

As we show in the following proposition, there exist examples of modules of constant Jordan types constructed as in Proposition 6.6 which are not middle terms of extensions of endotrivial modules as in Proposition 6.1.

Theorem 6.13. Let $E$ be an elementary abelian p-group of rank 3 and consider the $k E$-module $L=L_{\zeta_{1}, \zeta_{2}, \zeta_{3}}$ as in (16)), where $\left\{\zeta_{i}\right\}_{i=1,2,3} \subset \mathrm{H}^{2}(E, k)$ form a system of generators of $\mathrm{H}^{\bullet}(E, k)_{\text {red }}$. Then there does not exist a projective $k E$-modules $P$ such that $M=L \oplus P$ fits in a short exact sequence of the form

$$
0 \longrightarrow L^{\prime} \longrightarrow M \longrightarrow L^{\prime \prime} \longrightarrow 0
$$

with both $L^{\prime}, L^{\prime \prime}$ endotrivial modules.

Proof. Observe that $M$ has stable Jordan type 2[1], so that in any short exact sequence of the form (18) both $L^{\prime}, L^{\prime \prime}$ must have stable Jordan type 1[1]. We assume that such a short exact sequence exists and proceed to obtain a contradiction. First, by eliminating projective summands we may reduce to the case when both $L^{\prime}$ and $L^{\prime \prime}$ are projective-free. Since $E$ is a $p$-group, we have $P \cong(k E)^{t}$ for some $t \geq 0$. Since $L^{\prime}, L^{\prime \prime}$ have stable constant Jordan type 1[1], they are endotrivial by Theorem 5.6. If $p>2$, then we immediately conclude $L^{\prime} \cong \Omega^{2 n_{1}}(k), L^{\prime \prime} \cong \Omega^{2 n_{2}}(k)$ for some integers $n_{1}, n_{2}$ since $L^{\prime}, L^{\prime \prime}$ have stable Jordan type 1[1]. If $p=2$, then $\operatorname{dim} L^{\prime}+\operatorname{dim} L^{\prime \prime}=$ $\operatorname{dim} L+\operatorname{dim} P \equiv 2(\bmod \operatorname{dim} k E)$. Since $\operatorname{dim} \Omega^{n}(k) \equiv(-1)^{n}(\bmod \operatorname{dim} k E)$, we get that $L^{\prime}, L^{\prime \prime}$ must be even syzygies in the case $p=2$ as well. Thus, the sequence becomes

$$
0 \longrightarrow \Omega^{2 n_{1}}(k) \longrightarrow L \oplus(k E)^{t} \longrightarrow \Omega^{2 n_{2}}(k) \longrightarrow 0
$$

Let $\left\{g_{1}, g_{2}, g_{3}\right\}$ be group generators of $E$, and consider the subgroup $F=\left\langle g_{1}, g_{2}\right\rangle \subseteq$ $E$. The restriction of $\zeta_{3}$ to $F$ vanishes, so that

$$
L_{\mid F} \cong \Omega^{2}(k) \oplus L_{\mid F, \zeta_{1}, \zeta_{2}} \oplus(\text { proj })
$$

where $L_{\mid F, \zeta_{1}, \zeta_{2}}$ is constructed as in (16) with respect to the group $F$. By Lemma 6.12, $L_{\mid F, \zeta_{1}, \zeta_{2}} \cong \Omega^{4}(k)$ as a $k F$-module. Consequently,

$$
L_{\mid F} \cong \Omega^{2}(k) \oplus \Omega^{4}(k) \oplus(\operatorname{proj}) .
$$

Restricting (19) to $F$ and eliminating the projective summands at the ends, we obtain an exact sequence of $k F$-modules

$$
0 \longrightarrow \Omega^{2 n_{1}}(k) \longrightarrow \Omega^{2}(k) \oplus \Omega^{4}(k) \oplus(\operatorname{proj}) \longrightarrow \Omega^{2 n_{2}}(k) \longrightarrow 0
$$


By performing a shift and eliminating excess projectives, we get the sequence of $k F$-modules

$$
0 \longrightarrow \Omega^{2 n_{1}-2 n_{2}}(k) \longrightarrow \Omega^{4-2 n_{2}}(k) \oplus \Omega^{2-2 n_{2}}(k) \oplus(\operatorname{proj}) \stackrel{\theta}{\longrightarrow} k \longrightarrow 0
$$

where $\theta$ restricts to $\theta_{1}$ on $\Omega^{4-2 n_{2}}(k)$ and to $\theta_{2}$ on $\Omega^{2-2 n_{2}}(k)$. Since the kernel of $\theta$ has stable Jordan type 1[1], at least one of $\theta_{1}, \theta_{2}$ does not factor through a projective module when restricted to any $\pi$-point of $F$. Hence, Proposition 6.3 implies that $n_{2} \leq 2$. By the same argument applied to the other end of Sequence (21), we have that $2 n_{1}-2 n_{2}$ cannot be less than $2-2 n_{2}$ (i.e., $n_{1} \geq 1$ ). We further observe that if there were a non-trivial projective summand in the middle term of (21), then $\theta$ restricted to the projective summand would be surjective, and, hence, the kernel would have stable Jordan type different from 1[1]. It follows that there is no projective summand in the middle term of (21). Hence, we get $p^{3}\left(\left|4-2 n_{2}\right|+\left|2-2 n_{2}\right|\right)+2=\operatorname{dim}\left(\Omega^{4-2 n_{2}}(k) \oplus \Omega^{2-2 n_{2}}(k)\right)=\operatorname{dim} \Omega^{2 n_{1}-2 n_{2}}(k)+1=$ $p^{3}\left(\left|2 n_{1}-2 n_{2}\right|\right)+2$ by Lemma 6.9. Hence,

$$
\left|2-n_{2}\right|+\left|1-n_{2}\right|=\left|n_{1}-n_{2}\right|
$$

We conclude that $n_{1}+n_{2}=3$ when $n_{2} \leq 1$. Moreover, $n_{1}$ and $n_{2}$ must have different parity.

We consider two cases:

(I) $n_{2} \geq 0$, and

(II) $n_{2}<0$.

Case I: $n_{2} \geq 0$. We first show that the middle term of the sequence (19) does not have a projective summand, that is $t=0$.

The sequence (19) represents a cohomology class in $\widehat{\mathrm{H}}^{2 n_{2}-2 n_{1}+1}(E, k)$ which we denote by $\eta$. Restriction of (19) to any $\pi$-point of $E$ has the form

$$
0 \longrightarrow n[p]+1[1] \longrightarrow(n+m)[p]+2[1] \longrightarrow m[p]+1[1] \longrightarrow 0
$$

This sequence of $\mathbb{Z} / p \mathbb{Z}$-modules is necessarily split. Hence, $\eta$ vanishes upon restriction to any $\pi$-point of $E$.

Applying $\operatorname{Hom}_{\text {stmod }(k E)}(-, k)$ to the short exact sequence (19), viewed as a distinguished triangle in $\operatorname{stmod}(k E)$, we obtain a long exact sequence

$$
\cdots \longrightarrow \widehat{\operatorname{Ext}}_{k E}^{-1}\left(\Omega^{2 n_{1}}(k), k\right) \stackrel{\delta}{\longrightarrow} \widehat{\operatorname{Ext}}_{k E}^{0}\left(\Omega^{2 n_{2}}(k), k\right) \longrightarrow \widehat{\operatorname{Ext}}_{k E}^{0}(L, k) \longrightarrow \cdots
$$

which is equivalent to

$$
\cdots \longrightarrow \mathrm{H}^{2 n_{1}-1}(E, k) \stackrel{\cdot \eta}{\longrightarrow} \mathrm{H}^{2 n_{2}}(E, k) \longrightarrow \widehat{\operatorname{Ext}}_{k E}^{0}(L, k) \longrightarrow \cdots
$$


The rank of the free summand $(k E)^{t}$ in the middle term of the sequence (19) equals the dimension of the kernel of the map $\widehat{\operatorname{Ext}}_{k E}^{0}\left(\Omega^{2 n_{2}}(k), k\right) \longrightarrow \widehat{\operatorname{Ext}}_{k E}^{0}(L, k)$. Hence, it also equals the dimension of the image of the connecting homomorphism $\delta$, which is multiplication by $\eta$, a cohomology class of degree $\operatorname{deg} \eta=2 n_{2}-2 n_{1}+1$. Therefore, to show that $t=0$ we need to show that multiplication by $\eta$ on $\mathrm{H}^{2 n_{1}-1}(E, k)$ is trivial.

If $2 n_{2}-2 n_{1}+1>0$ then for $p>2$ the fact that $\eta$ vanishes upon restriction to any $\pi$-point implies that $\eta$ is divisible by the product $\eta_{1} \eta_{2} \eta_{3}$ where $\eta_{1}, \eta_{2}, \eta_{3}$ are the nilpotent generators in degree 1 of $\mathrm{H}^{*}(E, k)$. Thus, the multiplication by $\eta$ of any odd-dimensional class of positive degree is zero. Hence, the image of $\cdot \eta$ on $\mathrm{H}^{2 n_{1}-1}(E, k)$ is trivial since $2 n_{1}-1 \geq 1$. If $p=2$ then the only class which vanishes upon restriction to any $\pi$-point is the zero class. We conclude that $t=0$ in this case.

Now assume that $2 n_{2}-2 n_{1}+1<0$. Since cohomology of $E$ is not periodic, multiplication by $\eta$ on $\mathrm{H}^{-\operatorname{deg} \eta}(E, k)$ is trivial $([4,2.2])$. Since any monomial in $\mathrm{H}^{2 n_{1}-1}(E, k)$ factors as a product of a class of degree $-\operatorname{deg} \eta=2 n_{1}-2 n_{2}-1$ and a class of degree $2 n_{2}$, we further conclude that multiplication by $\eta$ on $\mathrm{H}^{2 n_{1}-1}(E, k)$ is trivial. Hence, $t=0$ in this case as well.

We now compare the dimensions of the terms of the sequence (19) in which we take $t=0$. Note that $n_{1}$ and $n_{2}$ are both non-negative in the case that we are considering. We get

$6 p^{3}+2=\operatorname{dim} L=\operatorname{dim} \Omega^{2 n_{1}}(k)+\operatorname{dim} \Omega^{2 n_{2}}(k)=n_{1}\left(n_{1}+1\right) p^{3}+1+n_{2}\left(n_{2}+1\right) p^{3}+1$

using Lemma 6.9, Hence, we get the following equation on $n_{1}, n_{2}$ :

$$
n_{1}^{2}+n_{2}^{2}+n_{1}+n_{2}=6
$$

The only non-negative integer solutions are $(2,0)$ and $(0,2)$. This is impossible since $\left(n_{1}, n_{2}\right)$ must have the opposite parity by (22). Hence, we obtain a contradiction as desired, and the case $n_{2} \geq 0$ is finished.

Case II. We now consider the case of negative $n_{2}$. The projective summand $(k E)^{t}$ in the middle term of the sequence (19) can not be any bigger than a projective cover of $\Omega^{2 n_{2}}(k)$ as otherwise, kernel of the map $(k E)^{t} \longrightarrow \Omega^{2 n_{2}}(k)$ has a projective submodule which is then a direct summand of the right hand term of (19). By Lemma 6.10, $t \leq 2 n_{2}^{2}-n_{2}$. Hence,

$$
\begin{aligned}
\operatorname{dim} L & \geq \operatorname{dim} \Omega^{2 n_{2}}(k)+\operatorname{dim} \Omega^{2 n_{1}}(k)-p^{3}\left(2 n_{2}^{2}-n_{2}\right) \\
& =p^{3}\left(n_{2}^{2}-n_{2}\right)+p^{3}\left(n_{1}^{2}+n_{1}\right)+2-p^{3}\left(2 n_{2}^{2}-n_{2}\right)
\end{aligned}
$$

Simplifying, and using the fact that $n_{2}+n_{1}=3$, we get

$$
\begin{aligned}
\operatorname{dim} L & \geq p^{3}\left(n_{1}^{2}-n_{2}^{2}+3-n_{2}\right)+2 \\
& =p^{3}\left(3\left(n_{1}-n_{2}\right)+3-n_{2}\right)+2=p^{3}\left(3 n_{1}+3-4 n_{2}\right)+2
\end{aligned}
$$


Since $n_{1} \geq 1$, and $n_{2}<0$, we conclude that $\operatorname{dim} L \geq 10 p^{3}+2$. On the other hand, $\operatorname{dim} L=6 p^{3}+2$ by Lemma 6.9 and the definition of $L$, and we have a contradiction.

\section{CONSTRAint ON RANKS}

A consequence of our constructive techniques is a new proof of a special case of Macaulay's Generalized Principle Ideal Theorem. The point is that if the coefficients are in a field of finite characteristic, then we can represent homogeneous elements of a multivariable polynomial ring as elements in the cohomology ring of an elementary abelian $p$-group. We can represent a matrix of such elements as a map of modules over the group algebra. Specifically, we have the following

Theorem 7.1. (See Exercise 10.9 of [13]) Suppose that $k$ is an algebraically closed field. Fix integers $n, r$ and $d_{1}, \ldots, d_{r+1}$, with $n \geq 3, r \geq 2$, and $d_{j}>0$ for all $j$. Let $P=k\left[x_{1}, \ldots, x_{n}\right]$ be a polynomial ring in $n$ variables. Let $A=A\left(x_{1}, \ldots, x_{n}\right)$ be $a(r+1) \times r$ matrix with the property that every entry in column $i$ of $A$ is a homogeneous polynomial in $P$ of degree $d_{j}$ for all $j=1, \ldots, r+1$. Then there is some point $\alpha \in k^{n} \backslash\{0\}$ such that $A(\alpha)$ has rank less than $r$. Equivalently, the determinants of the $r \times r$ minors of $A$ (which are elements of $P$ ) have a common non-trivial zero.

Proof. Assume first that the characteristic of $k$ is $p>0$, as in the rest of the paper. Let $E$ denote an elementary abelian $p$-group of rank $n$. As recalled in (17), $\mathrm{H}^{*}(E, k)$ contains a polynomial subring $Q \cong k\left[\zeta_{1}, \ldots, \zeta_{n}\right]$, where the elements $\zeta_{i}$ are in degree 2 if $p>2$ and in degree 1 if $p=2$. For the purposes of the argument, we assume that $p>2$. The proof in the even characteristic case is very similar.

Let $A=\left(a_{i, j}\right)$ where for each $i$ and $j, a_{i, j}=a_{i, j}\left(x_{1}, \ldots, x_{n}\right)$ is a homogeneous polynomial of degree $d_{j}$. Then, $a_{i, j}\left(\zeta_{1}, \ldots, \zeta_{n}\right)$ is an element in $\mathrm{H}^{2 d_{j}}(E, k)$. Moreover, such an element is uniquely represented by a cocycle

$$
a_{i, j}^{\prime}\left(\zeta_{1}, \ldots, \zeta_{n}\right): \Omega^{2 d_{j}}(k) \longrightarrow k .
$$

Now we let $A^{\prime}$ be the map

$$
A^{\prime}: \bigoplus_{t=1}^{r+1} \Omega^{2 d_{t}}(k) \longrightarrow k^{r}
$$

whose matrix is $A^{\prime}=\left(a_{i, j}^{\prime}\left(\zeta_{1}, \ldots, \zeta_{n}\right)\right)$.

We proceed to prove the theorem by contradiction, observing that if $A(\alpha)$ had rank $r$ for all $\alpha \in k^{n} \backslash\{0\}$, then, because $k$ is algebraically closed (so that the $k$ rational points of $\Pi(E)$ are dense), the kernel $L$ of $A^{\prime}$ would be a module of constant Jordan type (with stable Jordan type 1[1]) as in Theorem 6.6. By Theorem 5.6, $L$ 
is an endotrivial module. Hence, $L \cong \Omega^{2 m}(k)+(\operatorname{proj})$ for some $m$. Since $\bigoplus_{t=1}^{r+1} \Omega^{2 d_{t}}(k)$ does not have projective summands, we conclude that $L \cong \Omega^{2 m}(k)$.

As in the proof of Theorem 6.13, we can use a dimension argument to ascertain the value of $m$. Let $H \subset E$ be an elementary abelian subgroup of rank 2 . Restricting to $H$ and eliminating the projective summand in $L$ and in the domain of $A^{\prime}$, we get that the dimension of the projective-free part of $L \downarrow_{H}$ is precisely $p^{2} \sum_{i=1}^{r+1} d_{i}+1$ by Lemma 6.9 (4). Consequently, by the same lemma $m=\sum_{i=1}^{r+1} d_{i}$.

Now let $H^{\prime} \subset E$ be an elementary abelian $p$-subgroup of rank 3 , and let $L^{\prime}$ be the projective-free part of the restriction of $L$ to $H^{\prime}$. Since $L^{\prime} \simeq \Omega^{2 m}(k)$ as $H^{\prime}$-modules, Lemma 6.9 (5) implies that

$$
\operatorname{dim} L^{\prime}=p^{3} m(m+1)+1=p^{3}\left(\sum_{i=1}^{r+1} d_{i}\right)\left(\sum_{i=1}^{r+1} d_{i}+1\right)+1
$$

On the other hand, $L^{\prime}$ is the projective-free part of the kernel of the map $A^{\prime}$ restricted to $H^{\prime}$. Applying Lemma 6.9)(4) to compute the dimension of the $H^{\prime}$-module $\bigoplus_{t=1}^{r+1} \Omega^{2 d_{i}}(k)$ we get

$$
\operatorname{dim} L^{\prime}=p^{3} \sum_{t=1}^{r+1} d_{i}\left(d_{i}+1\right)+1 .
$$

As all $d_{i}>0$, the formula (24) clearly yields a greater value than (25). Thus, we get a contradiction.

Now, we consider a field of characteristic 0 , still denoted $k$. Let $R \subset k$ be the ring finitely generated over $\mathbb{Z}$ by the coefficients of the (homogeneous polynomial) entries of $A$. The $r+1$ determinants of the $r \times r$ minors of $A$ define a closed subscheme $Z$ of the projective scheme $\mathbb{P}_{R}^{n-1}$. By the preceding argument for fields of positive characteristic, $Z$ intersects each geometric fiber of $\mathbb{P}_{R}^{n-1} \longrightarrow$ Spec $R$ above a point of $\operatorname{Spec} R$ with positive residue characteristic.

Observe that the geometric points of Spec $R$ whose residue characteristics are positive are dense in Spec $R$. Hence, the image of $Z \subset \mathbb{P}_{R}^{n-1}$ in Spec $R$ is both closed and dense and thus all of $\operatorname{Spec} R$. We conclude that $Z$ intersects every geometric fiber of $\mathbb{P}_{R}^{n-1} \longrightarrow$ Spec $R$, including that given by $R \longrightarrow k$.

\section{Auslander Reiten Components}

Our objective in this section is to show how the Auslander-Reiten theory of almost split sequences can be used to construct modules having certain specific constant Jordan types. One of our main results is that if one module in a component of the 
Auslander-Reiten quiver of $k G$ has constant Jordan type and if $k$ is perfect, then every module in the component has constant Jordan type.

We begin this section by briefly recalling a few basic facts concerning the AuslanderReiten theory of almost split sequences, sometimes called Auslander-Reiten sequences. We refer the reader to [1] or [3, I.4] for more detailed accounts.

Suppose that $A$ is a finite dimensional algebra over the field $k$. A sequence of A-modules

$$
0 \longrightarrow L \stackrel{\zeta}{\longrightarrow} M \stackrel{\gamma}{\longrightarrow} N \longrightarrow 0
$$

is an almost split sequence if $M$ and $N$ are indecomposable, the sequence is not split, and one of the following two equivalent conditions holds:

(1) Any $A$-module map $\sigma: X \longrightarrow N$ which is not a splittable epimorphism, admits a factorization $\sigma=\gamma \theta$ for some homomorphism $\theta: X \longrightarrow M$.

(2) Any $A$-module map $\sigma: L \longrightarrow Y$ which is not a splittable monomorphism, admits a factorization $\sigma=\theta \zeta$ for some homomorphism $\theta: M \longrightarrow Y$.

(see [1, V.1]). If $M$ is an indecomposable non-projective $A$-module, then the almost split sequence of $A$-modules ending in $M$ is unique up to isomorphism and has the form

$$
0 \longrightarrow \tau M \longrightarrow B \longrightarrow M \longrightarrow 0
$$

where $\tau=\mathrm{D} \circ \mathrm{Tr}$ is the translation operator on the set of isomorphism classes of indecomposable non-projective $A$-modules defined as the composition of the transpose and duality functors (see [1, VII.1]). Similarly, there is a unique up to isomorphism almost split sequence starting with an indecomposable non-injective module $L$. If $A$ is a symmetric algebra (in particular, if $A=k G$ for a finite group $G$ ), then $\tau=\Omega^{2}$. We give description of $\tau$ in the case of any finite group scheme $G$ in the proof of Lemma 8.6.

Let $L, M$ be indecomposable $A$-modules. A map $\gamma: L \longrightarrow M$ is an irreducible map if the following two conditions hold:

(a) $\gamma$ is neither a splittable monomorphism nor a splittable epimorphism

(b) for any factorization $\gamma=\mu \nu$ either $\mu$ is a splittable monomorphism (has a right inverse) or $\nu$ is a splittable epimorphism (has a left inverse)

(see [1, V.5]). Irreducible maps are closely related to almost split sequences as the following lemma indicates.

Lemma 8.1. ([1, V.5.3]) Let $0 \longrightarrow L \stackrel{\zeta}{\longrightarrow} M \stackrel{\gamma}{\longrightarrow} N \longrightarrow 0$ be an almost split sequence, and let $M=\bigoplus_{i=1}^{r} M_{i}$ where each $M_{i}$ is an indecomposable module. Then the maps $\zeta_{i}: L \longrightarrow M_{i}$ and $\gamma_{i}: M_{i} \longrightarrow N$ that make up $\zeta$ and $\gamma$ are irreducible maps. Moreover, every irreducible map occurs in such a way in some almost split sequence. 
The next result shows that irreducible maps originating in projective modules, and consequently, almost split sequences with projective summands in the middle term have a very special form. We now take the algebra $A$ to be the group algebra $k G$ for a finite group scheme $G$. Since $k G$ is a Frobenius algebra, projective modules are injective and vice versa.

Lemma 8.2. ([1, V.5.5]) Let $G$ be a finite group scheme, and $P$ be an indecomposable projective $k G$-module. Then, up to scalar multiple, the only irreducible map originating at $P$ is the quotient map $P \longrightarrow P / \operatorname{soc}(P)$. Consequently, the only almost split sequence in which $P$ occurs is the almost split sequence ending in $P / \operatorname{soc}(P)$. This sequence has the form

$$
0 \longrightarrow \operatorname{rad}(P) \longrightarrow P \oplus \operatorname{rad}(P) / \operatorname{soc}(P) \longrightarrow \operatorname{rad}(P) / \operatorname{soc}(P) \longrightarrow 0
$$

For a finite group scheme $G$, the stable Auslander-Reiten quiver of $G$, denoted $\Gamma_{G}^{s}$, is a valued quiver whose vertices are isomorphism classes of indecomposable nonprojective $k G$-modules, and whose arrows are induced by irreducible morphisms (see [1, VII.1,4]). Namely, if there is an irreducible map $M \rightarrow N$ for non-projective indecomposable modules $M, N$, then there is an arrow $[M] \longrightarrow[N]$ in the AuslanderReiten quiver of $G$. By Lemma 8.1 , there is an arrow $[M] \longrightarrow[N]$ if and only if there exists an almost split sequence $0 \longrightarrow \tau N \longrightarrow \widetilde{M} \longrightarrow N \longrightarrow 0$ such that $M$ is an indecomposable direct summand of $\widetilde{M}$. A (stable) Auslander-Reiten component of an indecomposable non-projective module $M$ is a connected component of the Auslander-Reiten quiver $\Gamma_{G}^{s}$ containing the vertex $[M]$. In addition, the AuslanderReiten quiver is equipped with the translation automorphism $\tau: \Gamma_{G}^{s} \longrightarrow \Gamma_{G}^{s}$ induced by the translation operator $\tau$.

An Auslander-Reiten component $\Theta$ of $\Gamma_{G}^{s}$ has the form

$$
\Theta \simeq \mathbb{Z}\left[\vec{\Delta}_{\Theta}\right] / H
$$

where $\vec{\Delta}_{\Theta}$ is a directed tree, and $H \subset \operatorname{Aut}\left(\mathbb{Z}\left[\vec{\Delta}_{\Theta}\right]\right)$ is a group acting "admissibly" on $\vec{\Delta}_{\Theta}$ (see [1, VII.4] for definitions and [3, I.4.15.6] for the structure isomorphism). The underlying non-directed tree $\Delta_{\Theta}$ is uniquely determined by the component $\Theta$ and $\Theta$ is said to have tree class or type of $\Delta_{\Theta}$.

Later in the section we use the following result, which is due to Erdmann [14] in the case of finite groups. For arbitrary finite group schemes, there are similar results of Farnsteiner [16], [17].

Theorem 8.3. 14] Suppose that $G$ is a finite group and that $M$ is a $k G$-module which lies in a block of wild representation type. Then the Auslander-Reiten component of $M$ has tree class $A_{\infty}$.

The tree class $A_{\infty}$ tells us a lot about the structure of the almost split sequences. The vertices of a stable Auslander-Reiten component of such a tree class are obtained 
by iterated applications of the translation operator $\tau$ to a distinguished collections of vertices which correspond to isomorphism classes of non-projective indecomposable modules $X_{0}, X_{1}, X_{2}, \ldots$ such that $X_{0}$ is the beginning vertex and for each $i \geq 0$ there is an irreducible map $X_{i} \rightarrow X_{i+1}$. That is, we have a tree

$$
\Delta: \quad X_{0} \longrightarrow X_{1} \longrightarrow \cdots \longrightarrow X_{i} \longrightarrow X_{i+1} \longrightarrow \cdots
$$

such that the component is isomorphic to $\mathbb{Z}[\vec{\Delta}] / H$ as in (27). An almost split sequence involving modules in the component has the form

$$
0 \longrightarrow \tau^{n+1} X_{i} \longrightarrow \tau^{n+1} X_{i+1} \oplus \tau^{n} X_{i-1} \longrightarrow \tau^{n} X_{i} \longrightarrow 0
$$

for some $n$ as long as $i \geq 1$. Here, $\tau^{0}$ is the identity operator, $\tau^{-1}=\operatorname{Tr} \circ D$, etc. On the boundary of the component $(i=0)$, the almost split sequence has the form

$$
0 \longrightarrow \tau^{n+1} X_{0} \longrightarrow \tau^{n+1} X_{1} \oplus P \longrightarrow \tau^{n} X_{0} \longrightarrow 0
$$

where $P$ might be zero or might be an indecomposable projective module (see [1, VII.4]). However, as stated in Lemma 8.2, each indecomposable projective module occurs in a unique almost split sequence (which is not necessarily in the component under consideration).

Before showing how almost split sequences can be used to generate indecomposable modules of constant Jordan type, we need the following technical lemma. We fix some notation which differs from standard notation in the case of finite groups: In what follows, let $A \subset B$ be rings, and $M$ be a left $A$-module. Then

$$
\operatorname{Coind}_{\mathrm{A}}^{\mathrm{B}} \mathrm{M}=\mathrm{B} \otimes_{\mathrm{A}} \mathrm{M}
$$

Let $G$ be a finite group scheme, and $\alpha_{K}: K[t] / t^{p} \longrightarrow K G$ be a $\pi$-point. We denote by $K\left\langle\alpha_{K}(t)\right\rangle$ the subalgebra of $K G=K G_{K}$ generated by $\alpha_{K}(t)$.

Lemma 8.4. Let $G$ be a finite group scheme and let $\alpha_{K}: K[t] / t^{p} \rightarrow K G$ be a $\pi$-point of $G$. Let $N$ be a finite dimensional $K[t] / t^{p}$-module which is not projective and set $M=$ Coind $_{\mathrm{K}\left\langle\alpha_{\mathrm{K}}(\mathrm{t})\right\rangle}^{\mathrm{KG}} \mathrm{N}$. Then

$$
\Pi\left(G_{K}\right)_{M}=\left\{\left[\alpha_{K}\right]\right\} \subset \Pi\left(G_{K}\right) .
$$

Proof. If $\Pi\left(G_{K}\right)_{M}$ were empty, then $M$ would be projective, and, hence, injective. On the other hand, the Eckmann-Shapiro Lemma ([3, I.2.8.4]) would enable us to then conclude that $\operatorname{Ext}_{G_{K}}^{*>0}(M, K)=\operatorname{Ext}_{K\left\langle\alpha_{K}(t)\right\rangle}^{*>0}(N, K)=0$ which would contradict our assumption that $N$ is not projective. Thus, $\Pi\left(G_{K}\right)_{M}$ is non-empty.

Let $U_{K} \subset G_{K}$ be a unipotent abelian subgroup scheme through which $\alpha_{K}$ factors. The proof of [21, 4.12] which is stated for induction rather than coinduction implies that

$$
\Pi\left(G_{K}\right)_{M} \subset \operatorname{im}\left\{\Pi\left(\mathrm{U}_{\mathrm{K}}\right) \rightarrow \Pi\left(\mathrm{G}_{\mathrm{K}}\right)\right\}
$$

so that we may assume that $G_{K}=U_{K}$ is a unipotent abelian finite group scheme over $K$. 
After possibly replacing $K$ by some purely inseparable extension which does not change the space $\Pi\left(G_{K}\right)_{M}$, we may by [28, 14.4] assume that $K G_{K}$ is isomorphic (as an algebra) to $K\left[T_{1}, \ldots, T_{n}\right] /\left(T_{1}^{p^{e_{1}}}, \ldots, T_{n}^{p^{e_{n}}}\right)$ for suitable choice of $n, e_{1}, \ldots, e_{n}$. Let $t_{i}=T_{i}^{p^{e_{i}-1}}$, and recall that any $\pi$-point $\beta_{L}: L[t] / t^{p} \rightarrow L G_{K}$ must send $t$ to a sum of monomials in $T_{1}, \ldots, T_{n}$ at least one of which is a non-linear scalar multiple of some $t_{i}$ and each of which are divisible by some (possibly varying) $t_{i}$. By a change of generators, we may arrange that $\alpha_{K}(t)=t_{1}+p(T)$ where each monomial of the polynomial $p(T)$ is a non-scalar multiple of some $t_{i}$.

In order to verify that $\left[\beta_{L}\right] \notin \Pi\left(G_{K}\right)_{M}$ for $\left[\beta_{L}\right] \neq\left[\alpha_{K}\right]$, we may choose a representative $\beta_{L}$ of $\left[\beta_{L}\right]$ which is linear in the $t_{i}$ 's. Assuming $\left[\beta_{L}\right] \neq\left[\alpha_{K}\right]$, we may change generators once again so that $\alpha_{K}(t)$ retains the form $t_{1}+p(T)$ as above and $\beta_{L}(t)=t_{2}$. The condition that $\left[\beta_{L}\right] \notin \Pi\left(G_{K}\right)_{M}$ is equivalent to the condition that $\beta_{L}^{*}\left(M_{L}\right)$ is free. Clearly, it suffices to assume that $N$ is indecomposable of the form $K[t] / t^{i}, i<p$. Then $M_{L} \cong L\left[T_{1}, \ldots, T_{n}\right] /\left(t_{1}^{p}, \ldots, t_{n}^{p},\left(t_{1}+p(T)\right)^{i}\right)$ which is free over $L\left\langle t_{2}\right\rangle$ with a monomial basis $\left\{T_{1}^{j_{1}}, T_{2}^{j_{2}}, \ldots, T_{n}^{j_{n}}\right\}$, where $0 \leq j_{1}<i p^{e_{1}-1}, 0 \leq j_{2}<$ $p^{e_{2}-1}, 0 \leq j_{3}<p^{e_{3}} \ldots 0 \leq j_{n}<p^{e_{n}}$.

We now prove a local restriction result for almost split exact sequences of $k G$ modules.

Proposition 8.5. Let $G$ be a finite group scheme such that the dimension of $\Pi(G)$ is at least 1 , and let $M$ be an indecomposable non-projective $k G$-module of constant Jordan type. Assume that one of the following conditions hold: either $M$ is absolutely indecomposable or $k$ is perfect. Consider the almost split sequence of $k G$-modules

$$
\mathcal{E}: 0 \longrightarrow N \longrightarrow B \longrightarrow M \longrightarrow 0 \text {. }
$$

Then for any $\pi$-point $\alpha_{K}: K[t] / t^{p} \longrightarrow K G, \alpha_{K}^{*}\left(\mathcal{E}_{K}\right)$ is a split short exact sequence of $K[t] / t^{p}$-modules.

Proof. If $M_{K}$ is decomposable, write $M_{K} \cong \oplus M_{K}^{i}$ as a direct sum of indecomposable $K G_{K}$-modules. Since in this case $k$ is perfect, Theorem [24, 3.8] implies that the almost split sequence $\mathcal{E}_{K}$ is a direct sum of almost split sequences

$$
\mathcal{E}_{K}^{i}: 0 \longrightarrow N_{K}^{i} \longrightarrow B_{K}^{i} \longrightarrow M_{K}^{i} \longrightarrow 0
$$

Thus, it suffices to prove that $\alpha_{K}^{*}\left(\mathcal{E}_{K}^{i}\right)$ is split for each $i$. Hence, we may assume that $M_{K}$ is neither projective nor decomposable, and that

$$
\mathcal{E}_{K}: 0 \longrightarrow N_{K} \longrightarrow B_{K} \longrightarrow M_{K} \longrightarrow 0
$$

is an almost split sequence of $K G_{K}$-modules. 
Let $\widetilde{M}_{K}=\operatorname{Coind}_{\mathrm{K}\left\langle\alpha_{\mathrm{K}}(\mathrm{t})\right\rangle}^{\mathrm{KG}}\left(\alpha_{\mathrm{K}}^{*}\left(\mathrm{M}_{\mathrm{K}}\right)\right)$. We have a commutative diagram

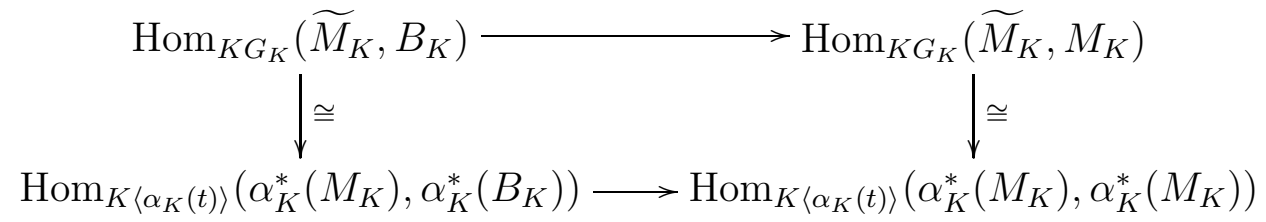

where the vertical arrows are isomorphisms by the Eckmann-Shapiro Lemma. If $\alpha_{K}^{*}\left(\mathcal{E}_{K}\right)$ were not split, then the lower horizontal arrow of (28) and thus also the upper horizontal arrow of (28) would not be surjective. On the other hand, the defining property of almost split sequences would then imply that $\widetilde{M}_{K}$ must have $M_{K}$ as a direct summand. If so, then $\Pi\left(G_{K}\right)_{M_{K}} \subset \Pi\left(G_{K}\right)_{\widetilde{M}_{K}}$. Since $M_{K}$ is a non-projective module of constant Jordan type, we have $\Pi\left(G_{K}\right)_{M_{K}}=\Pi\left(G_{K}\right)$, and, hence, the support of $M_{K}$ has dimension at least 1 . Since $\Pi\left(G_{K}\right)_{\widetilde{M}_{K}}$ consists of only 1 point by Lemma 8.4, we obtain a contradiction.

Lemma 8.6. Let $G$ be a finite group scheme, and $M$ be an indecomposable nonprojective finite-dimensional $k G$-module. Then $M$ is a module of constant Jordan type if and only if $\tau M$ is a module of constant Jordan type. Moreover, if $M$ is a module of constant Jordan type then the stable Jordan types of $M$ and $\tau M$ are the same.

Proof. Since $k G$ is self-injective, the translation operator $\tau$ is isomorphic to $\mathcal{N} \circ \Omega^{2}$ where $\mathcal{N}$ is the Nakayama functor defined as

$$
\mathcal{N}=\operatorname{Hom}_{k G}(-, k G)^{\#}
$$

(see [1, IV.3]).

The Heller shift $\Omega^{2}$ preserves the property of being of constant Jordan type, and, moreover, preserves the stable Jordan type of a module of constant Jordan type (1.8). Hence, to show that $\tau$ preserves modules of constant Jordan type, we need to demonstrate this for the Nakayama functor.

By [23, I.8.12] we can exhibit a character $\delta$ of $G$ with the property that $k G^{\#} \otimes$ $k_{\delta} \simeq k G$ is a two-sided $k G$-isomorphism, where $k_{\delta}$ is the one-dimensional $k G$ representation with $k G$ acting trivially on the right and by the character $\delta$ on the left. Hence, we get the following isomorphisms of $k G$-modules

$$
\begin{gathered}
\operatorname{Hom}_{k G}(M, k G) \simeq \operatorname{Hom}_{k G}\left(M, k G^{\#} \otimes k_{\delta}\right) \simeq \operatorname{Hom}_{k G}\left(M \otimes k_{\delta}^{\#}, k G^{\#}\right) \simeq \\
\operatorname{Hom}_{k G}\left(M \otimes k_{\delta}^{\#}, \operatorname{Hom}_{k}(k G, k)\right) \simeq \operatorname{Hom}_{k}\left(M \otimes k_{\delta}^{\#}, k\right)=M^{\#} \otimes k_{\delta}
\end{gathered}
$$

Hence,

$$
\mathcal{N}(M)=\operatorname{Hom}_{k G}(M, k G)^{\#} \simeq\left(M^{\#} \otimes k_{\delta}\right)^{\#} \simeq M \otimes k_{\delta}^{\#}
$$

as $k G$-modules. Let $M$ be a module of constant Jordan type. Since the module $k_{\delta}$ is 1-dimensional, it is of trivial constant Jordan type. Hence, the module $\mathcal{N}(M) \simeq$ 
$M \otimes k_{\delta}^{\#}$ is also of constant Jordan type by Corollary 4.3. Moreover, the Jordan type of $M \otimes k_{\delta}^{\#}$ is the same as that of $M$.

To show that $\tau M$ being of constant Jordan type implies that $M$ is of constant Jordan type, we merely observe that the operator $\tau$ has an inverse given by $\Omega^{-2} \circ$ $\mathcal{N}^{-1}$. Hence, if $\tau M$ is of constant Jordan type then so is $M$.

The following theorem asserts that whether or not an indecomposable $k G$-module $M$ has constant Jordan type is a function of the connected components of the stable Auslander-Reiten quiver of $k G$.

Theorem 8.7. Let $G$ be a finite group scheme, and let $M$ be an indecomposable non-projective module of constant Jordan type. Let $\Theta$ be a component of the stable Auslander-Reiten quiver of $k G$ containing the module $M$. Assume that one of the following conditions hold: either all vertices of $\Theta$ are absolutely indecomposable, or $k$ is perfect. Then for any $[N] \in \Theta$, the module $N$ has constant Jordan type.

Proof. We first consider the case when $\operatorname{dim} \Pi(G)=0$. By Theorem $[3.4$, $\Pi(G)$ consists of one point. Hence, any module is tautologically a module of constant Jordan type.

We may therefore assume that $\operatorname{dim} \Pi(G) \geq 1$. Let $[N]$ be any predecessor of $[M]$ in the stable quiver component $\Theta$. Then there exists an almost split sequence

$$
\mathcal{E}: 0 \longrightarrow \tau M \longrightarrow B \longrightarrow M \longrightarrow 0
$$

such that $N$ is a direct summand of $B$. By Lemma 8.6, $\tau M$ is a module of constant Jordan type. Let $\alpha_{K}: K[t] / t^{p} \rightarrow K G_{K}$ be a $\pi$-point. By Proposition $8.5, \alpha_{K}^{*}\left(\mathcal{E}_{K}\right)$ splits. Thus, $\alpha_{K}^{*}\left(B_{K}\right)=\alpha^{*}\left(\tau M_{K}\right) \oplus \alpha^{*}\left(M_{K}\right)$. We conclude that $B$ is a module of constant Jordan type, so that Theorem 3.7 implies that $N$ has constant Jordan type.

Now let $[N]$ be any successor of $[M]$, i.e., there is an arrow $[M] \longrightarrow[N]$. By $[1$, V.1.12] and [1, V.5.3], there is an arrow $[\tau N] \longrightarrow[M]$. Applying the argument above to $\tau N$ and $M$, we conclude that $\tau N$ has constant Jordan type. By Lemma 8.6 , $N$ has constant Jordan type.

Since $\Theta$ is connected, the argument is finished by induction.

To prove the following "realization of constant types" result, we appeal to the work of K. Erdmann [14] in the case of finite groups and that of R. Farnsteiner [16], [17] for arbitrary finite group schemes (see 8.3). Namely, a result of [15] (see also [17]) following earlier work of Webb [29] asserts that if $k G$ has wild representation type then the Auslander-Reiten component of the trivial module has a very restricted form. Results of Erdmann and Farnsteiner assert that under hypotheses specified in the theorem below, the Auslander-Reiten component of the trivial module must have type $A_{\infty}$. 
Theorem 8.8. Let $G$ be a finite group scheme (over $k$ algebraically closed) satisfying one of the following conditions: either $G$ is a finite group which has p-rank at least 2 and whose Sylow p-subgroup is not dihedral or semi-dihedral or $\Pi(G)$ has dimension at least 2. Then for any $n$ there exists an indecomposable module of stable constant Jordan type $n[1]$.

Proof. By [14, 2] in the case of finite groups and [17, 3.3] for arbitrary finite group schemes, our assumptions imply that Auslander-Reiten quiver of the component containing the trivial module must have type $A_{\infty}$.

Let $V_{n}$ be an indecomposable module representing the $n^{\text {th }}$ vertex of the tree underlying the component containing the trivial module. The bottom vertex has label 0. By Proposition 8.7, $V_{n}$ has constant Jordan type for every $n$. Let $\underline{a}_{n}$ be the stable Jordan type of $V_{n}$. Proposition 8.5 and Lemma 8.6 imply that the middle term $B_{n}$ of the almost split sequence

$$
0 \longrightarrow \tau V_{n} \longrightarrow B_{n} \longrightarrow V_{n} \longrightarrow 0
$$

has stable constant Jordan type $2 \underline{a}_{n}$.

Since the tree class is $A_{\infty}, V_{1}$ must be the only non-projective indecomposable summand of the middle term of the almost split sequence for $V_{0}$ :

$$
0 \longrightarrow \tau V_{0} \longrightarrow B_{0} \longrightarrow V_{0} \longrightarrow 0
$$

Hence, $\underline{a}_{1}=2 \underline{a}_{0}$. The middle term of the almost split sequence for $V_{1}$

$$
0 \longrightarrow \tau V_{1} \longrightarrow B_{1} \longrightarrow V_{1} \longrightarrow 0
$$

has two indecomposable non-projective summands, one of which is isomorphic to $\tau V_{0}$. Hence, the other summand has stable constant Jordan type $2 \underline{a}_{1}-\underline{a}_{0}=3 \underline{a}_{0}$. Proceeding by induction, we see that the module which represents the $n^{\text {th }}$ node in this $A_{\infty}$ tree has stable constant Jordan type $\underline{a}_{n}=n \underline{a}_{0}$.

We immediately conclude that $k$ must be at the bottom node since the stable Jordan type of $k$ is 1[1]. Hence, $\underline{a}_{0}=1[1]$. Therefore, $V_{n}$ is an indecomposable module of stable constant Jordan type $n[1]$.

\section{Questions And Conjectures}

We offer a few broad questions as well as specific conjectures which provide challenges for further investigation.

Question 9.1. For a given finite group scheme $G$, what Jordan types are realized as the Jordan type of finite dimensional $k G$-modules with constant Jordan type?

Certainly, there are constraints as the following examples illustrate. 
Example 9.2. Let $G$ be a quasi-elementary abelian group scheme, $G=\mathbb{G}_{a(s)} \times E$ with $E$ and elementary abelian $p$-group of rank $r$ and $p>2$. Then any $k G$-module $M$ of constant Jordan type of stable type 1[1] is endotrivial, and hence of the form $\Omega^{i}(k)$ [12]. The Jordan type of such a module has the form $1[1]+m p^{r+s-1}[p]$ for some $m \geq 0$ (see Lemma 6.9).

Example 9.3. We verify that there does not exist a finite dimensional $k E$-module of constant Jordan type $[2]+[p]$ for $p>3$ for $E$ an elementary abelian $p$-group of rank 2 , which the reader can see as very limited evidence for Conjecture 9.5. Suppose $V$ is such a $k E$-module and write $k E=k[x, y] /\left(x^{p}, y^{p}\right)$. Consider the $k$-vector space basis $u, x u, v, x v, x^{2} v, \ldots, x^{p-1} v$ for $V$.

We will show that some linear combination $y-b x$ satisfies $(y-b x)^{p-1} V=0$, so that the Jordan form associated to $y-b x$ has no block of size $p$. Observe that $y(v)$ written in our given basis has coefficient 0 for $v$ because $y$ is nilpotent and only $v$ in our basis satisfies $x^{p-1} v \neq 0$. Second, suppose that $y(v)$ has coefficient $b$ for $x v$ and consider $y-b x$. Then once again $(y-b x)(v)$ has coefficient 0 for $v$ and by construction coefficient 0 for $x v$. Let us replace $y$ by $y-b x$, so that $y(v) \in \operatorname{span}\left\{u, x u, x^{2} v, \ldots, x^{p-1} v\right\}$.

If we apply $y$ to the given basis, the only basis element with the property that $y$ applied to it can have non-zero coefficient for $u$ is $u$ itself, since any of the other basis elements would have to be annihilated by $x^{2}$ and thus in the image of $x$. Since $y$ is nilpotent, we conclude that $y(u) \in \operatorname{span}\left\{x u, x^{2} v, \ldots, x^{p-1} v\right\}$ so that $y^{2}(u) \in$ $\operatorname{span}\left\{x^{4} v, \ldots, x^{p-1} v\right\}$.

Thus, $y^{3} u$ and $y^{3} v$ are both contained in $x^{4} V$, and we conclude that $y^{p-1}\left(x^{i} v\right)=$ $0=y^{p-1}\left(x^{i} u\right)$.

The challenge of Question 9.1 seems more interesting if we work stably, so that we identify two Jordan types $\underline{a}=a_{p}[p]+\cdots+a_{1}[1], \underline{b}=b_{p}[p]+\cdots+b_{1}[1]$ provided that $a_{i}=b_{i}, i \neq p$.

Question 9.4. For which finite group schemes $G$ is every stable Jordan type the Jordan type of a finite dimensional $k G$-module of constant Jordan type?

The following is a specific conjecture would be a step towards answering the previous question.

Conjecture 9.5. Let $E$ be an elementary abelian p-group of rank $\geq 2$, with $p>3$. Then there does not exist a finite dimensional $k E$-module of stable constant Jordan type [2].

Andrei Suslin has formulated the following intriguing question whose affirmative answer would in particular verify the preceding conjecture.

Question 9.6. Let $E$ be an elementary abelian p-group of rank 2, with $p>3$. Let $M$ be a $k E$-module with constant Jordan type $\sum_{i} a_{i}[i]$ and let $i$ be an integer, $1<i<p$. Is it the case that if $a_{i} \neq 0$, then either $a_{i+1} \neq 0$ or $a_{i-1} \neq 0$ ? 
Of course, if Conjecture 9.5 is valid, then it follows that there is no module of stable constant Jordan type [2] for any finite group scheme $G$ containing a quasielementary subgroup scheme $H=\mathbb{G}_{a(r)} \times E$ such that the rank $s$ of $E$ plus $r$ is greater or equal to 2.

We can make many other "non-existence conjectures" such as the following. We recall (see Example 2.2) that for $E$ an elementary abelian $p$-group of rank $n \geq 2$ there exists a $k E$-module whose stable type is constant of type $1[2]+(n-1)[1]$

Conjecture 9.7. Let $E$ be an elementary abelian $p$-group of rank $n \geq 2$, with $p>3$. There does not exist a $k E$-module whose stable type is constant of type $1[2]+j[1]$ with $j \leq n-2$.

We next formulate questions of a more qualitative nature.

Definition 9.8. Let $G$ be a finite group scheme over a field $k$ (of characteristic $p>0$ ). We denote by $\mathcal{L}=\mathbb{N}^{p}$ the (additive) lattice of Jordan types over $k$. We denote by

$$
\mathcal{R}(G) \subset \mathcal{L}
$$

the sublattice of those Jordan types which can be realized as the Jordan types of $k G$-modules of constant Jordan type.

Question 9.9. For which finite group schemes $G$ is $\mathcal{L} / \mathcal{R}(G)$ finite? Among such finite group schemes, how does the invariant $\mathcal{L} / \mathcal{R}(G)$ behave?

For those finite group schemes $G$ for which $\mathcal{L} / \mathcal{R}(G)$ is infinite, can we give some interpretation of the rank of this quotient in more familiar terms?

In view of our discussion involving Auslander-Reiten almost split sequences, it seems of considerable interest to consider $\mathcal{I}(G)$ as defined below.

Definition 9.10. Let $G$ be a finite group scheme over a field $k$ (of characteristic $p>0$ ) and let $\overline{\mathcal{R}}(G) \subset \overline{\mathcal{L}}=\mathbb{N}^{p-1}$ denote the subset of those stable Jordan types realizable as the stable Jordan type of a finite dimensional $k G$-module of constant Jordan type. Further, let us denote by $\mathcal{I}(G) \subset \overline{\mathcal{R}}(G) \subset \overline{\mathcal{L}}=\mathbb{N}^{p-1}$ the subset of stable Jordan types which are the Jordan types of indecomposable $k G$-modules of constant Jordan type.

Question 9.11. For which $G$ is $\mathcal{I}(G)$ closed under addition?

Remark 9.12. If $G$ is the Klein four group, $G \simeq \mathbb{Z} / 2 \mathbb{Z} \times \mathbb{Z} / 2 \mathbb{Z}$, then the only non-projective indecomposable modules of constant Jordan type are Heller shifts of the trivial module $k$. Thus, for this choice of $G, \mathcal{I}(G)$ is not closed under addition.

One is tempted to ask many questions concerning how the realizability of modules of constant type behaves with respect to change of finite group scheme. We ask one such question. 
Question 9.13. For which $H \subset G$ does restriction induce a bijection from the set of Jordan types realized as Jordan types of $k G$-modules of constant Jordan type to the set of Jordan types realized as Jordan types of $k H$-modules of constant Jordan type?

10. APPENDIX: Decomposition of TEnsor PRoducts of $k[t] / t^{p}$-Modules

The purpose of this appendix is to derive a closed form for tensor products of $k[t] / t^{p}$-modules, presumably implicit in [27]. Here, we view $k[t] / t^{p}$ as a self-dual Hopf algebra; in other words, as the restricted enveloping algebra of the 1-dimensional $p$-restricted Lie algebra (with trivial $p$-restriction operator). Thus, the coproduct is given by the formula $t \mapsto 1 \otimes t+t \otimes 1$.

Let $V(\lambda)$ denote the simple restricted $s l_{2}$-module of highest weight $\lambda$ where $0 \leq$ $\lambda \leq p-1$. Let $e, h, f$ be the standard generators for $s l_{2}$. Let $\langle e\rangle \subset s l_{2}$ be the 1 -dimensional $p$-restricted Lie algebra generated by the element $e$. Hence, $u(\langle e\rangle) \simeq$ $k[t] / t^{p}$ is a Hopf subalgebra of $u\left(s l_{2}\right)$.

The $s l_{2}$-representation theory implies that

$$
V(\lambda) \downarrow_{u(\langle e\rangle)} \simeq[\lambda+1]
$$

as $k[t] / t^{p}$-modules. Hence, the tensor product formulas for indecomposable $k[t] / t^{p}$ modules follow from the tensor product formulas for simple $s l_{2}$-modules. Such formulas were obtained in [25] (see also [6]).

Proposition 10.1. (See [25, Lemma 2.3, 2.4].) Let $\{V(\lambda)\}_{0 \leq \lambda \leq p-1}$ be the collection of simple restricted $\mathrm{sl}_{2}$-modules.

- If $0 \leq \mu \leq \lambda \leq p-2$ and $\lambda+\mu \leq p-1$ then

$$
V(\lambda) \otimes V(\mu) \simeq V(\lambda-\mu) \oplus V(\lambda-\mu+2) \oplus \ldots \oplus V(\lambda+\mu)
$$

- If $0 \leq \mu \leq \lambda \leq p-2$ and $\lambda+\mu \geq p$ then

$$
V(\lambda) \otimes V(\mu) \simeq V(\lambda-\mu) \oplus V(\lambda-\mu+2) \oplus \ldots \oplus V(2(p-2)-\lambda-\mu)+(\text { proj })
$$

where the projective summand has dimension $\lambda+\mu-(p-2)$.

Restricting the tensor decompositions for the simple restricted $s l_{2}$-modules $V(\lambda)$ to the indecomposable $k[t] / t^{p}$-modules $[i]$ via the formula (29) we obtain the following formulas.

Corollary 10.2. Let $[i]$ be an indecomposable $k[t] / t^{p}$-module of dimension $i$ for $1 \leq i \leq p$. Then

$$
[i] \otimes[j]= \begin{cases}{[j-i+1]+[j-i+3]+\ldots+[j+i-3]+[j+i-1]} & \text { if } j+i \leq p \\ {[j-i+1]+\ldots+[2 p-1-i-j]+(j+i-p)[p]} & \text { if } j+i>p .\end{cases}
$$


We conclude this appendix with the analogous formula for the tensor product of indecomposable $k C_{p}$-modules where $C_{p}$ is a cyclic group of order $p$. A subtlety here is that even though the module categories for $k C_{p}$ and the algebra $k[t] / t^{p}$ with the coproduct $t \mapsto 1 \otimes t+t \otimes 1$ are equivalent, the tensor product structure comes from two different coproducts. Nonetheless, the tensor multiplicities turn out to be the same.

Corollary 10.3. Let $[i], 1 \leq i \leq p$, be indecomposable $k C_{p}$-modules. Then the decomposition of the tensor product $[i] \otimes[j]$ into indecomposable $k C_{p}$-modules is given by the formulas as in Corollary 10.2.

Proof. By [21, 4.5] the tensor product of any two $k C_{p}$-modules $M, N$ is isomorphic as $k[t] / t^{p}$-module to the tensor product $M \otimes N$ using the coproduct $t \mapsto 1 \otimes t+t \otimes 1$. The statement now follows from Corollary 10.2 .

\section{REFERENCES}

[1] M. Auslander, I. Reiten, and S. Smalo, Representation Theory of Artin Algebras, Cambridge Studies in Advanced Mathematics 36, 1995.

[2] V. Basev, Representations of the group $\mathbb{Z}_{2} \times \mathbb{Z}_{2}$ in a field of characteristic 2, (Russian) Dokl. Akad. Nauk. USSR 141 (1961), 1015- 1018.

[3] D. Benson, Representations and Cohomology, vols. I and II, Cambridge University Press, 1991.

[4] D. Benson and J. Carlson, Products in negative cohomology, J. Pure Appl. Algebra, 82 (1992), 107-129.

[5] A. J. Berkson, The u-algebra of a restricted Lie algebra is Frobenius, Proc. Amer. Math. Soc. 15 (1964), 14-15.

[6] G. Benkart, J. Osborn, Representations of rank one Lie algebras of characteristic p, Lect. Notes Math. 933 (1982), 1-37.

[7] J. Carlson, The varieties and the cohomology ring of a module, J. Algebra 85 (1983), 104-143.

[8] J. Carlson, The variety of an indecomposable module is connected, Invent. Math. 77 (1984), 291-299.

[9] J. Carlson and J. Thévenaz, Torsion endo-trivial modules, Algebras and Rep. Theory, 3 (2000), 303-335.

[10] J. Carlson and J. Thévenaz, The classification of endo-trivial modules, Invent. Math. 158 (2004), 389-411.

[11] J. Carlson, L. Townsley, L. Valero-Elizondo, M. Zhang, Cohomology rings of finite groups, Kluwer, 2003.

[12] E. C. Dade, Endo-permutation modules over p-groups, I, II, Ann. Math. 107 (1978), 459-494, 108 (1978), 317-346.

[13] D. Eisenbud, Commutative Algebra with a View Towards Algebraic Geometry, Springer-Verlag, 1995.

[14] K. Erdmann, On Auslander-Reiten components of group algebras, J. Pure Appl. Algebra 104 (1995), 149-160.

[15] K. Erdmann, M. Holloway, N. Snashall, Ø. Solberg, R. Taillefer, Support varieties for selfinjective algebras, K-Theory 33 (2004), 67-87.

[16] R. Farnsteiner, On the Auslander-Reiten quiver of an infinitesimal group, Nagoya Math. J. 160 (2000), 103-121. 
[17] R. Farnsteiner, Support spaces and Auslander-Reiten components, to appear in Contemp. Math.

[18] E. Friedlander, J. Pevtsova, Representation-theoretic support spaces for finite group schemes, Amer. J. Math. 127 (2005), 379-420.

[19] E. Friedlander, J. Pevtsova, Erratum: Representation-theoretic support spaces for finite group schemes, Amer. J. Math. 128 (2006), 1067-1068.

[20] E. Friedlander, J. Pevtsova, П-supports for modules for finite group schemes, to appear in Duke. Math. J.

[21] E. Friedlander, J. Pevtsova, A Suslin Generic and Maximal Jordan types, Invent. Math. 168 (2007), 485-522.

[22] A. Heller, I Reiner, Indecomposable representations, Illinois J. Math 5 (1961), 314-323.

[23] J. Jantzen, Representations of Algebraic Groups, Academic Press, 1987.

[24] S. Kasjan, Auslander-Reiten sequences under base field extension, Proc. Amer. Math. Soc. 128 (2000), no. 10, 2885-2896.

[25] A. Premet, The Green ring of a simple three-dimensional Lie p-algebra (Russian) Izv. Vyssh. Uchebn. Zaved. Mat. (1991), no. 10, 56-67; translation in Soviet Math. (Iz. VUZ) 35 (1991), no. $10,51-60$.

[26] A. Suslin, E. Friedlander, C. Bendel, Infinitesimal 1-parameter subgroups and cohomology, J. Amer. Math. Soc. 10 (1997) 693-728.

[27] B. Srinivasan, The modular representation ring of a cyclic p-group, Proceedings of the London Math Soc. XIV, no. 36 (1964), 677-688.

[28] W. Waterhouse, Introduction to affine group schemes, Graduate Texts in Mathematics, 66 Springer-Verlag, New York-Berlin, 1979.

[29] P. J. Webb, The Auslander-Reiten quiver of a finite group, Math. Zeit. 179 (1982), 97-121.

Department of Mathematics, University of Georgia, Athens, GA

E-mail address: jfc@math.uga.edu

Department of Mathematics, Northwestern University, Evanston, IL

E-mail address: eric@math.northwestern.edu

Department of Mathematics, University of Washington, Seattle, WA

E-mail address: julia@math.washington.edu 